\title{
21. LITHOLOGIC AND CHEMICAL STRATIGRAPHY AT DEEP SEA DRILLING PROJECT SITES 417 AND 418
}

\author{
M. F. J. Flower, ${ }^{1}$ W. Ohnmacht, ${ }^{2}$ P. T. Robinson, ${ }^{3}$ G. Marriner, ${ }^{4}$ and H.-U Schmincke ${ }^{2}$
}

\begin{abstract}
Chemical compositions of basalts, detailed lithologic data, and stable magnetic inclinations are used to define an eruptive stratigraphy for the basement sections at Holes 417D and 418A. A maximum of 25 eruptive units, comprising three major sequences, are recognized in the 365 meter-thick section at Hole 417D, and a maximum of 54 eruptive units grouped into seven eruptive cycles are defined in the 544 meters penetrated at Hole 418A. The rocks in Hole $417 \mathrm{~A}$ are too altered to permit detailed stratigraphic analysis.

The freshness of the rocks at Holes 417D and 418A suggests that the basement was sealed off shortly after formation by sedimentation and growth of secondary phases in the lava pile. Several horizons marked by the presence of breccia, by changes in magnetic inclinations and chemical compositions, and by increased alteration are believed to reflect major quiescent intervals in the eruptive record. These define a pattern of episodic eruption, with each episode possibly derived from a separate magma reservoir system. Variations in downhole magnetic inclinations suggest that crustal deformation occurs during each eruptive cycle, and that oscillatory lateral migration of the active zone may occur before a crustal section is complete.

Chemical variation of the basalts is not extensive and is dominated by fractionation of olivine, plagioclase, clinopyroxene, and spinel. Phenocryst movement during eruption and flow is fairly complex, but accumulation of plagioclase at or near the site of initial crystallization is a widespread phenomenon. Most magmas had reached a low-pressure cotectic stage by the time of eruption, but relict clinopyroxenes indicate an earlier stage of fractionation.

Dikes were intersected in the lower part of Hole 418A and correspond chemically to flows 130 meters higher in the section. These may represent the top of an underlying dike complex, originally forming nearsurface feeder conduits, but now buried by subsequent eruptions.
\end{abstract}

\section{INTRODUCTION}

This paper presents chemical analyses of 205 whole-rock samples from Sites 417 and 418, drilled in Cretaceous basement in the western Atlantic Ocean on DSDP Legs 51, 52, and 53 (Figure 1).

We have integrated these data with shipboard observations of lithologic and magnetic stratigraphy and chemical variation of basaltic glass (Byerly and Sinton, this volume). Our main aim is to establish a chemical stratigraphy of the basement sections in Holes 417D and 418A in order to inter-

\footnotetext{
${ }^{1}$ Dept. of Mineral Sciences, Museum of Natural History, Smithsonian Institution, Washington, D.C. at time of cruise. Present address: 25 Willes Road, London NW5, England.

${ }^{2}$ Institut für Mineralogie der Ruhr-Universität, D-4630 Bochum, West Germany.

${ }^{3}$ Department of Geological Sciences, University of California, Riverside, California.

${ }^{4}$ Department of Geology, Bedford College, London NW1, England.
}

pret igneous processes at the Cretaceous mid-ocean spreading axis. We proceed by defining eruptive units and major quiescent intervals, and then examine the relation between eruptive chronology and compositional variation of the basalts. From these relationships, the sampled section is interpreted in terms of dynamic crustal construction processes.

\section{ANALYTICAL TECHNIQUES}

Eight samples from Hole 417A, 45 from Hole 417D, and 152 from Hole $418 \mathrm{~A}$ were analyzed for major elements at Bochum, West Germany, while a selection of 71 samples from Hole 418A were analyzed for $\mathrm{Sr}, \mathrm{Y}, \mathrm{Zr}, \mathrm{Rb}$, and $\mathrm{Nb}$ at Bedford College, London, England. Samples were carefully selected for analysis from representative portions of all flow units identified onboard ship so as to represent all major lithologic types. A few extremely altered specimens were analyzed, particularly from Hole 417A. These were not representative of original basalt compositions and served only to monitor alteration processes. 


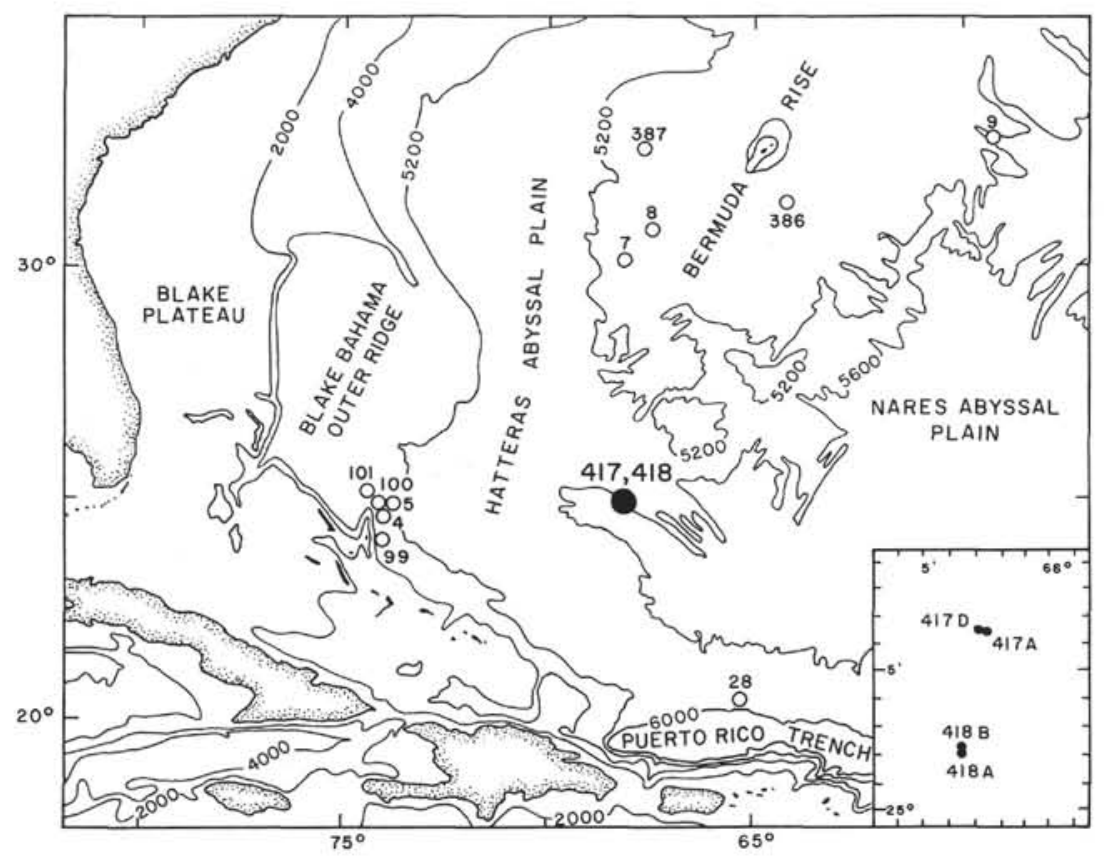

Figure 1. Location map of Sites 417 and 418 at the southern end of Bermuda Rise, western Atlantic Ocean.

Major oxides were determined by X-ray fluorescence, using a Philips PW 1410 under conditions as tabulated:

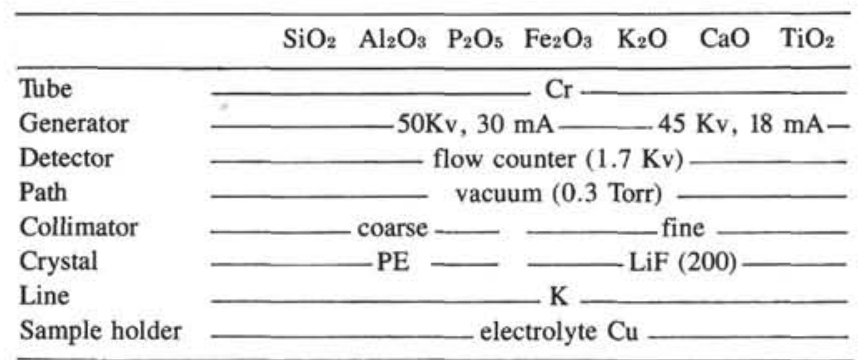

Glass disks were prepared of: (a) international standards; (b) three Columbia River basalt samples (courtesy of T. L. Wright); (c) interlaboratroy comparison specimen, DSDP Sample 417A-44-3, 58-68 cm; and (d) DSDP Leg 51, 52, and 53 rock powders. The following proportions were used: $6.39 \mathrm{~g} \mathrm{Na}_{2} \mathrm{~B}_{4} \mathrm{O}_{7}$ dried at $550^{\circ} \mathrm{C}$ (Spectromelt B10, Merck $6304)$ and $0.71 \mathrm{~g}$ rock powder dried at $1000^{\circ} \mathrm{C}$. Unpolished disks were analyzed after confirmation that grinding and polishing made no difference in the analyses. The raw data were corrected for background and drift. Least-squares calibration curves were computed for each oxide, using the data of Abbey (1973) multiplied by loss-on-ignition (LOI) factors. LOI was determined on powders dried at $110^{\circ} \mathrm{C}$ for 24 hours. No corrections were made for $\mathrm{Ca}-\mathrm{P}$ spectral interferences, however; the error for $\mathrm{P}$ is thought to be negligible because unknowns and standards are similar in composition.

Determinations of $\mathrm{FeO}, \mathrm{Na}_{2} \mathrm{O}, \mathrm{MnO}, \mathrm{CO}_{2}$, and $\mathrm{H}_{2} \mathrm{O}^{+}$ were made potentiometrically, by atomic absorption, by coulometric titration (CTA-5 analyzer), and by coulometric Karl Fischer titration (Aquatest analyzer). Analytical results are given in Table 1 . The interlaboratory comparison sample 15 is included in Table 1A as "Bochum Sample No. 250."
Table 2 gives the DSDP designations for all specimens analyzed.

Trace elements were analyzed with a Philips PW 1212 XRF spectroscope on pressed powder pellets. Data for samples analyzed and standards are given in Table 1B.

\section{LITHOLOGY}

Basement recovery at Sites 417 and 418 averaged between 70 and 80 per cent and in some intervals exceeded 95 per cent. The high recovery, coupled with the freshness of most material cored from Holes 417D and 418A, makes possible an unexpectedly detailed study of the nature of oceanic Layer 2A. Drilled sequences at both Holes 417D and $418 \mathrm{~A}$ appear to be typical of eruptive ocean crust, consisting of interlayered pillowed and massive basalt with lesser intercalations of breccia and sediment. Major differences from younger Atlantic basement (drilled on Legs 37, 45,46 , and 49) are the comparative scarcity of interlayered sediment and the greater abundance of breccia. Very low sedimentation rates at the time of crust formation (Orr and Miles, this volume) probably account for the lack of sediment, whereas the higher proportion of breccia probably reflects the higher degree of induration of the old crust. The high recovery enables us to identify all or most igneous cooling units, to correctly interpret their contacts and to identify highly altered layers. From these observations it is possible for the first time to make a realistic stratigraphic interpretation of the upper part of Layer 2.

As a first step in such an interpretation, we have subdivided the cored sequences into detailed lithologic subunits. A sub-unit is defined as a single igneous cooling unit, a breccia zone, or a sedimentary intercalation, regardless of any genetic association between contiguous sequences. In igneous sequences, cooling unit boundaries were identified from glassy selvages or from abrupt changes in grain size. In general, a unit boundary was inferred where a cooling 
break traversed the core but not where it was vertical. Cooling units defined in this way include individual pillows with an average vertical thickness of about 0.5 meters (Robinson et al., this volume) and massive flows ranging from about 2 to 10 meters in thickness.

Breccia sequences and intercalated sediments are somewhat more difficult to interpret. Breccias may be "incipient" or comparatively well developed, and may vary in thickness from a few centimeters to about 1.5 meters. Some are hyaloclastites, clearly related to the process of pillow formation; others, termed "polymict" in this paper, are made up of angular clasts of variable lithology and composition, and probably represent accumulations of submarine talus. This type of breccia probably makes up lithologic Unit 6A in Hole 418A and Unit 9A in Hole 417D (Figures 2 and 3). Occasionally, a thick sequence of incipient breccia is bounded above and below by highly brecciated zones, possibly reflecting large fallen blocks buried by rubble and lava eruptions. However, distinctions between breccia types are difficult to make and for the purposes of unit definition we have assumed there to be only one lithologic type. Recognition of intercalated sediment is also difficult in many cases. Thick veins or patches of nonfossiliferous carbonate and smectite, with or without pyrite, often occur between cooling units, but it is not clear whether these are altered sediments or secondary mineral deposits. The euxinic bottom-water conditions during crust formation and the mixed (silica-carbonate) nature of these materials suggest that many are lithified sediment. Thus, we have interpreted such assemblages occurring between cooling units and exceeding $5 \mathrm{~cm}$ in thickness as sedimentary intercalations.

Using the criteria outlined above, we have recognized over 400 lithologic sub-units in Hole 417D and over 600 sub-units in Hole 418A. For each hole, units are numbered consecutively downhole and assigned an approximate subbottom depth computed from the core log. A listing of these units is available on request. For the purposes of this paper, however, we have used only the major lithologic units defined by the shipboard parties.

\section{MAGNETIC STRATIGRAPHY}

The magnetic stratigraphy at Holes $417 \mathrm{D}$ and $418 \mathrm{~A}$ is complex, in common with most other basement sections drilled in the Atlantic Ocean. Stable inclinations are variable and often differ markedly from predicted dipole values. At Hole 418A, five downhole polarity reversals have been observed or inferred (Rigotti et al., this volume). Shipboard scientists of Legs 51,52 , and 53 do not agree on the interpretation of the magnetic data (Rigotti; Levi; Bleil and Smith; all, this volume), but it seems unlikely that secular changes of the magnetic field (Hall, in press; Watkins and Walker, 1977) can explain all of the downhole variation. We believe that the close coincidence of magnetic, lithologic, and chemical boundaries in the sequences at Sites 417 and 418 reflects deformation of discrete blocks during crustal construction. Thus, the magnetic pattern may provide a record of dynamic processes in the overall tectonic evolution of the active zone. However, interpretation of drill core data is complicated by the lack of sample control compared to subaerial lava flows, and the difficulty of testing tilting or rotation models. For this reason, we have attempted to iden- tify magnetic "units" in the core, each unit reflecting a basalt sequence with closely similar stable inclinations. These units have been plotted against depth (Figures 2 and 3 ), together with shipboard lithologic divisions, in order to provide a framework in which to examine the downhole chemical variations. For a single cooling unit (pillow or massive unit) a single natural remanent magnetization (NRM) inclination is assumed to be representative of the whole unit. The validity of this assumption is supported by the uniformity of inclinations in relatively thick massive units with several magnetic data points. Some scatter of inclinations is observed in massive units near the base of Hole $418 \mathrm{~A}$, but those are believed due to the intrusion of dikes with polarities opposite to those of the intruded flows. Hyaloclastite and polymict breccias may be distinguished according to the range of inclinations measured for individual clasts: the former have inclinations similar to the enclosing lavas, while the latter have a nearly random distribution of inclinations.

\section{BASALT PETROGRAPHY}

Shipboard lithologic units for each site provide an accurate stratigraphic breakdown of the core. The updated lithologic subdivisions for Holes 417D and 418A are given in Figures 2 and 3 . Before proceeding to a stratigraphic synthesis of rock chemistry and other properties, we shall give a brief summary of the basalt petrography. A more detailed description of basalt lithology is given in the Site Chapters in Part I of this volume.

\section{Hole 417D}

The 13 lithologic units identified in Hole 417D consist of pillowed basalt, massive basalt, and several thick horizons of basalt breccia. Plagioclase (An70-80) is the main phenocryst phase in all basalts, but olivine and clinopyroxene are also present in small amounts. The plagioclase is relatively fresh and occurs as single-zoned grains and as glomerocrysts. Many crystals have abundant glass inclusions, often arranged in concentric bands. Clinopyroxene phenocrysts are present in most pillow basalts as fresh rounded crystals or glomerocrysts. Rounded clinopyroxene phenocrysts also occur at the base of the massive flow comprising lithologic Unit 1A. Phenocrystic olivine occurs in all lithologic units and is invariably altered to carbonate, with or without smectite. Dark red-brown spinel is present in lithologic Unit 4, either in the matrix or as microcrysts enclosed by clinopyroxene. The spinel crystals are rounded and have no oxidation rims. Subophitic plagioclase/clinopyroxene clots, with or without altered olivine, occur throughout the section, being distributed sporadically down to lithologic Unit 4 and widely below that level.

Groundmass textures in the pillow basalts range from quench to intersertal or intergranular. In massive units the groundmass has an "ophimottled"' texture in which subophitic patches of plagioclase and clinopyroxene are partly surrounded by glass. Groundmass minerals in both pillowed and massive units are plagioclase, clinopyroxene, altered olivine, and minor iron oxides.

Vesicles or amygdules are abundant only in the uppermost parts of some massive flows (lithologic Units 2 and 3) 
Major-Oxide Analyses of Whole-Rock Samples From DSDP Holes 417A, 417D, and 418A

\begin{tabular}{|c|c|c|c|c|c|c|c|c|c|c|c|c|c|c|c|c|c|c|c|c|c|c|c|c|}
\hline \multirow{2}{*}{$\begin{array}{l}\text { Bochum } \\
\text { Sample No. }\end{array}$} & \multicolumn{7}{|c|}{ Hole 417A } & \multicolumn{17}{|c|}{ Hole 417D } \\
\hline & 110 & 120 & 160 & 180 & 210 & 220 & 240 & 250 & 1010 & 1020 & 1040 & 1060 & 1080 & 1110 & 1120 & 1140 & 1150 & 1170 & 1180 & 1185 & 1210 & 1240 & 1275 & 1280 \\
\hline $\mathrm{SiO}_{2}$ & 46.90 & 47.12 & 48.53 & 47.05 & 47.03 & 48.55 & 49.06 & 49.25 & 46.99 & 48.28 & 47.72 & 48.67 & 47.30 & 48.05 & 48.91 & 49.62 & 47.76 & 47.54 & 48.17 & 48.10 & 47.81 & 49.36 & 48.41 & 47.93 \\
\hline $\mathrm{TiO}_{2}$ & 1.28 & 1.27 & 1.32 & 1.32 & 1.29 & 1.45 & 1.39 & 1.42 & 1.54 & 1.43 & 1.32 & 1.28 & 1.32 & 1.32 & 1.36 & 1.34 & 1.28 & 1.35 & 1.35 & 1.41 & 1.28 & 1.49 & 1.38 & 1.35 \\
\hline $\mathrm{Al}_{2} \mathrm{O}_{3}$ & 18.05 & 16.89 & 16.92 & 16.63 & 16.14 & 16.93 & 16.47 & 16.33 & 17.54 & 17.06 & 16.39 & 16.78 & 16.84 & 17.44 & 17.39 & 17.21 & 17.46 & 16.90 & 16.44 & 18.42 & 16.15 & 17.92 & 16.69 & 16.88 \\
\hline $\mathrm{Fe}_{2} \mathrm{O}_{3}$ & 4.05 & 3.48 & 3.57 & 3.88 & 4.99 & 3.81 & 2.92 & 3.59 & 5.80 & 4.39 & 5.14 & 3.36 & 4.49 & 4.62 & 5.36 & 4.43 & 5.22 & 4.55 & 3.92 & 5.11 & 2.99 & 5.56 & 4.22 & 4.63 \\
\hline $\mathrm{FeO}^{\circ}$ & 2.93 & 4.71 & 5.88 & 4.91 & 4.79 & 4.96 & 7.19 & 6.64 & 3.56 & 5.00 & 5.16 & 6.57 & 4.76 & 4.37 & 4.23 & 4.48 & 4.75 & 4.78 & 5.22 & 4.16 & 5.80 & 4.07 & 5.88 & 5.37 \\
\hline $\mathrm{MnO}$ & 0.12 & 0.16 & 0.16 & 0.17 & 0.19 & 0.17 & 0.17 & 0.16 & 0.11 & 0.17 & 0.16 & 0.16 & 0.16 & 0.14 & 0.09 & 0.13 & 0.12 & 0.15 & 0.15 & 0.14 & 0.17 & 0.11 & 0.16 & 0.18 \\
\hline $\mathrm{MgO}$ & 5.06 & 6.69 & 6.78 & 6.17 & 5.86 & 6.58 & 6.53 & 6.92 & 5.29 & 7.03 & 6.54 & 6.75 & 5.75 & 5.36 & 5.62 & 5.16 & 5.37 & 5.90 & 5.71 & 5.43 & 5.73 & 5.57 & 6.11 & 6.14 \\
\hline $\mathrm{CaO}$ & 14.17 & 14.38 & 13.27 & 14.45 & 14.19 & 13.26 & 12.13 & 12.47 & 11.42 & 11.85 & 12.76 & 12.65 & 13.97 & 13.48 & 11.52 & 13.11 & 13.16 & 13.22 & 13.67 & 12.48 & 14.80 & 10.28 & 12.58 & 12.73 \\
\hline $\mathrm{Na}_{2} \mathrm{O}$ & 2.05 & 2.91 & 2.57 & 2.08 & 2.69 & 2.01 & 1.94 & 2.03 & 2.40 & 2.15 & 2.06 & 2.00 & 2.38 & 2.12 & 2.27 & 2.11 & 2.22 & 2.05 & 2.15 & 2.41 & 1.95 & 2.40 & 2.36 & 2.01 \\
\hline $\mathrm{K}_{2} \mathrm{O}$ & 0.95 & 0.09 & 0.03 & 0.18 & 0.33 & 0.03 & 0.07 & 0.08 & 0.55 & 0.07 & 0.27 & 0.05 & 0.06 & 0.29 & 0.38 & & 0.1 & 0.20 & 0.08 & 0.35 & 0.06 & 0.79 & 0.36 & 0.26 \\
\hline $\mathrm{P}_{2} \mathrm{O}_{5}$ & 0.12 & 0.15 & 0.16 & 0.15 & 0.15 & 0.16 & 0.15 & 0.14 & 0.17 & 0.14 & 0.16 & 0.15 & 0.16 & 0.17 & 0.15 & 0.16 & 0.15 & 0.16 & 0.16 & 0.17 & 0.15 & 0.15 & 0.13 & 0.15 \\
\hline $\mathrm{CO}_{2}$ & 2.16 & 1.48 & 0.38 & 1.38 & 1.46 & 0.43 & 0.13 & 0.13 & 1.93 & 0.84 & 1.18 & 0.18 & 1.78 & 1.15 & 0.46 & 0.61 & 0.83 & 1.50 & 1.61 & 0.75 & 1.98 & 0.34 & 0.67 & 0.95 \\
\hline $\mathrm{H}_{2} \mathrm{O}^{+}$ & 2.76 & 1.67 & 1.27 & 1.52 & 1.54 & 1.88 & 1.44 & 0.88 & 2.32 & 1.94 & 1.75 & 1.38 & 1.86 & 1.78 & 2.08 & 1.84 & 1.79 & 1.74 & 1.14 & 1.88 & 0.71 & 1.95 & 1.45 & 1.60 \\
\hline Sum & 100.60 & 101.00 & 100.84 & 99.89 & 100.65 & 100.22 & 99.59 & 100.04 & 99.62 & 100.35 & 100.61 & 99.98 & 100.83 & 100.29 & 99.82 & 100.25 & 100.26 & 100.04 & 99.77 & 100.81 & 99.58 & 99.99 & 100.40 & 100.18 \\
\hline $\mathrm{H}_{2} \mathrm{O}^{-}$ & 1.23 & 1.34 & 0.75 & 1.21 & 0.94 & 1.04 & 0.53 & 0.11 & 1.92 & 2.22 & 0.99 & 1.14 & 1.51 & 1.70 & 2.20 & 1.23 & 1.54 & 1.67 & 0.54 & 1.97 & 0.45 & 1.81 & 0.94 & 0.85 \\
\hline FeO Total & 6.57 & 7.84 & 9.09 & 8.40 & 9.28 & 8.39 & 9.82 & 9.87 & 8.78 & 8.95 & 9.78 & 9.59 & & 8.53 & 9.05 & 8.47 & 9.45 & 8.87 & 8.75 & 8.76 & 8.49 & 9.07 & 9.68 & 9.54 \\
\hline Mg No. & 60.90 & 63.32 & 60.14 & 59.77 & 56.09 & 61.35 & 57.37 & 58.65 & 54.94 & 61.38 & 57.49 & 58.74 & 56.93 & 55.98 & 55.67 & 55.22 & 53.49 & 57.36 & 56.91 & 55.64 & 57.72 & 55.40 & 56.09 & 56.57 \\
\hline
\end{tabular}

Note: $\mathrm{FeO}$ Total $=$ Total iron oxide expressed as $\mathrm{FeO} ; \mathrm{Mg} \mathrm{No} .=$ Magnesium number $=\mathrm{Mg} /\left(\mathrm{Mg}+\mathrm{Fe}^{+2}\right)$, assuming $\mathrm{Fe}^{+3}=0.1 \times \mathrm{Fe}^{+2}$.

TABLE 1A - Continued

\begin{tabular}{|c|c|c|c|c|c|c|c|c|c|c|c|c|c|c|c|c|c|c|c|c|c|c|c|c|}
\hline \multirow{2}{*}{$\begin{array}{c}\text { Bochum } \\
\text { Sample No. }\end{array}$} & \multirow[b]{2}{*}{1300} & \multirow[b]{2}{*}{1320} & \multirow[b]{2}{*}{1330} & \multirow[b]{2}{*}{1400} & \multirow[b]{2}{*}{1440} & \multirow[b]{2}{*}{1460} & \multirow[b]{2}{*}{1480} & \multirow[b]{2}{*}{1500} & \multirow[b]{2}{*}{1510} & \multicolumn{4}{|c|}{ Hole 417D - Continued } & \multirow[b]{2}{*}{1560} & \multirow[b]{2}{*}{1570} & \multirow[b]{2}{*}{1590} & \multirow[b]{2}{*}{1600} & \multirow[b]{2}{*}{1620} & \multirow[b]{2}{*}{1630} & \multirow[b]{2}{*}{1650} & \multirow[b]{2}{*}{1660} & \multirow[b]{2}{*}{1670} & \multirow[b]{2}{*}{1710} & \multirow[b]{2}{*}{1720} \\
\hline & & & & & & & & & & 1520 & 1530 & 1540 & 1550 & & & & & & & & & & & \\
\hline $\mathrm{SiO}_{2}$ & 47.10 & 46.83 & 48.53 & 48.96 & 47.19 & 47.91 & 48.11 & 48.07 & 48.99 & 46.94 & 48.34 & 48.81 & 47.90 & 46.59 & 47.82 & 47.64 & 48.73 & 47.70 & 49.47 & 46.39 & 47.31 & 47.13 & 48.46 & 47.98 \\
\hline $\mathrm{TiO}_{2}^{2}$ & 1.27 & 1.30 & 1.26 & 1.13 & 1.42 & 1.49 & 1.52 & 1.61 & 1.55 & 1.39 & 1.46 & 1.40 & 1.52 & 1.63 & 1.60 & 1.55 & 1.54 & 1.53 & 1.48 & 1.56 & 1.34 & 1.37 & 1.34 & 1.36 \\
\hline $\mathrm{Al}_{2} \mathrm{O}_{3}$ & 16.66 & 15.88 & 17.89 & 18.20 & 16.16 & 15.78 & 15.94 & 16.84 & 17.07 & 16.05 & 17.09 & 15.18 & 15.81 & 15.41 & 15.57 & 15.56 & 15.29 & 15.70 & 15.74 & 15.72 & 17.70 & 16.42 & 16.78 & 16.72 \\
\hline $\mathrm{Fe}_{2} \mathrm{O}_{3}$ & 3.41 & 4.29 & 3.72 & 3.77 & 4.75 & 4.74 & 4.92 & 4.94 & 5.10 & 5.66 & 4.39 & 4.23 & 5.13 & 4.23 & 5.00 & 4.86 & 4.16 & 4.14 & 4.76 & 6.80 & 4.36 & 4.11 & 4.40 & 4.44 \\
\hline $\mathrm{FeO}$ & & & & & 40 & 5.00 & 5.64 & 4.07 & 4.17 & 4. & 5.3. & 6.74 & 5.85 & 6.06 & 6.15 & & 7.20 & & 4.59 & 4.61 & 4.86 & 4.39 & 5.99 & 6.12 \\
\hline $\mathrm{MnO}$ & 0.19 & 0.19 & 0.16 & 0.16 & 0.15 & 0.16 & 0.20 & 0.12 & 0.14 & 0.13 & 0.16 & 0.17 & 0.18 & 0.23 & 0.22 & 0.20 & 0.20 & 0.18 & 0.15 & 0.18 & 0.17 & 0.16 & 0.16 & 0.17 \\
\hline $\mathrm{MgO}$ & & & & & & & & & & & & & & & 6.95 & & & & & & 6.02 & 6.94 & 6.22 & 5.92 \\
\hline $\mathrm{CaO}$ & 14.63 & 14.30 & 13.47 & 12.92 & 13.90 & 12.97 & 12.50 & 12.11 & 11.37 & 12.81 & 11.49 & 12.19 & 12.35 & 13.75 & 12.49 & 12.59 & 12.29 & 13.22 & 13.35 & 12.82 & 13.61 & 13.84 & 12.86 & 13.08 \\
\hline $\mathrm{Na}_{2} \mathrm{O}$ & & & & 1.87 & 1.98 & 2.66 & 2.03 & 2.47 & 2.7 & 2.50 & 2.4 & 2.34 & 2.28 & 2.34 & 2.41 & 2.28 & 2.2 & 2. & 2.5 & 2.41 & 2.40 & 2.43 & 2.21 & 2.16 \\
\hline $\mathrm{K}_{2} \mathrm{O}$ & 0.07 & 0.27 & 0.05 & 0.04 & 0.34 & 0.32 & 0.21 & 0.13 & 0.24 & 0.38 & 0.22 & 0.09 & 0.11 & 0.14 & 0.05 & 0.08 & 0.04 & 0.04 & 0.08 & 0.46 & 0.08 & 0.05 & 0.04 & 0.04 \\
\hline $\mathrm{P}_{2} \mathrm{O}_{5}$ & & 0.16 & & 0.14 & 0.17 & 0.18 & 0.17 & 0.17 & 0.15 & 0.17 & 0.1 & 0.1 & 0.1 & 0.20 & 0.17 & 0.16 & 0.1 & 0.1 & 0.1 & 0.18 & 0.14 & 0.14 & 0.13 & 0.15 \\
\hline $\mathrm{CO}_{2}$ & 1.85 & 1.78 & 0.87 & 0.39 & 1.98 & 1.34 & 0.96 & 1.22 & 0.67 & 1.51 & 1.2 & 0.4 & 0.55 & 1.55 & 0.46 & 0.38 & 0.2 & 0.7 & 0.3 & 1. & 1.2 & 1.70 & 0.40 & 0.52 \\
\hline $\mathrm{H}_{2} \mathrm{O}^{+}$ & 0.93 & 1.20 & 1.07 & 1.78 & 1.72 & 1.59 & 1.58 & 1.81 & 1.60 & 1.46 & 0.96 & 1.08 & 1.39 & 1.14 & 1.10 & 1.27 & 1.14 & 1.07 & 1.63 & 1.69 & 1.46 & 1.34 & 1.30 & 1.26 \\
\hline Sum & 100.89 & 100.35 & 100.66 & 100.25 & 100.15 & 100.56 & 100.08 & 100.60 & 100.83 & 99.79 & 100.30 & 99.80 & 100.25 & 99.90 & 99.99 & 99.44 & 100.62 & 100.18 & 100.86 & 100.22 & 100.70 & 100.02 & 100.29 & 99.92 \\
\hline $\mathrm{H}_{2} \mathrm{O}^{-}$ & 0.88 & 0.71 & 0.87 & 1.38 & 0.93 & 1.25 & 1.16 & 1.61 & 1.52 & 0.94 & 0.75 & 0.74 & 1.37 & 1.16 & 1.33 & 1.06 & 0.91 & 1.13 & 1.57 & 1.11 & 1.36 & 1.27 & 1.01 & 1.06 \\
\hline & 8.78 & 9.68 & & 7.75 & 8.67 & 9.27 & 10.07 & 8.52 & & & & & & 9.87 & 10.6 & 10.53 & & & & 10.73 & 8.78 & 8.09 & 9.95 & 10.12 \\
\hline Mg No. & 60.37 & 55.05 & 58.05 & 63.02 & 58.28 & 58.37 & 55.87 & 62.59 & 61.85 & 57.08 & 60.63 & 57.28 & 57.61 & 57.62 & 56.90 & 56.31 & 57.71 & 57.03 & 60.04 & 52.33 & 58.10 & 63.45 & 55.85 & 54.21 \\
\hline
\end{tabular}


TABLE 1A - Continued

\begin{tabular}{|c|c|c|c|c|c|c|c|c|c|c|c|c|c|c|c|c|c|c|c|c|c|c|c|c|}
\hline \multirow{2}{*}{$\begin{array}{c}\text { Bochum } \\
\text { Sample No. }\end{array}$} & \multicolumn{5}{|c|}{ Hole 417D - Continued } & \multicolumn{19}{|c|}{ Hole 418A } \\
\hline & 1730 & 1740 & 1760 & 1780 & 1790 & 10 & 15 & 20 & 30 & 40 & 50 & 60 & 70 & 80 & 90 & 100 & 103 & 106 & 110 & 120 & 130 & 136 & 140 & 150 \\
\hline $\mathrm{SiO}_{2}$ & 48.56 & 48.87 & 48.41 & 49.72 & 48.52 & 46.74 & 48.98 & 48.41 & 48.29 & 47.76 & 47.12 & 49.10 & 48.77 & 49.40 & 49.19 & 48.37 & 50.18 & 49.44 & 48.47 & 48.91 & 49.48 & 49.27 & 49.45 & 49.12 \\
\hline $\mathrm{TiO}_{2}$ & 1.36 & 1.41 & 1.32 & 1.39 & 1.25 & 1.09 & 1.22 & 1.22 & 1.20 & 1.17 & 1.10 & 1.15 & 1.10 & 1.10 & 1.15 & 1.11 & 1.09 & 1.07 & 1.03 & 1.03 & 1.06 & 1.05 & 1.09 & 1.12 \\
\hline $\mathrm{Al}_{2} \mathrm{O}_{3}$ & 16.56 & 17.01 & 16.78 & 16.05 & 16.93 & 17.18 & 18.27 & 17.50 & 17.88 & 17.70 & 16.89 & 17.03 & 16.09 & 16.30 & 16.69 & 16.11 & 16.63 & 16.30 & 16.65 & 16.68 & 16.41 & 16.78 & 16.78 & 16.33 \\
\hline $\mathrm{Fe}_{2} \mathrm{O}_{3}$ & 4.02 & 4.02 & 3.87 & 3.90 & 3.01 & 4.64 & 3.83 & 4.07 & 3.88 & 3.88 & 3.89 & 3.96 & 3.57 & 3.00 & 3.26 & 3.05 & 3.01 & 3.06 & 2.69 & 2.88 & 2.98 & 2.09 & 3.42 & 3.24 \\
\hline $\mathrm{FeO}$ & 6.41 & 5.40 & 6.33 & 6.74 & 6.95 & 4.16 & 4.79 & 4.50 & 3.87 & 3.80 & 4.44 & 5.00 & 5.77 & 6.53 & 5.88 & 6.10 & 6.28 & 6.24 & 5.94 & 6.20 & 6.32 & 6.91 & 5.38 & 6.29 \\
\hline $\mathrm{MnO}$ & 0.17 & 0.22 & 0.18 & 0.17 & 0.19 & 0.11 & 0.12 & 0.12 & 0.14 & 0.11 & 0.14 & 0.12 & 0.15 & 0.17 & 0.16 & 0.17 & 0.16 & 0.16 & 0.18 & 0.17 & 0.16 & 0.16 & 0.15 & 0.17 \\
\hline $\mathrm{MgO}$ & 6.41 & 6.48 & 6.57 & 6.81 & 7.23 & 6.59 & 6.86 & 7.13 & 7.20 & 6.96 & 6.73 & 7.08 & 7.44 & 7.34 & 6.95 & 6.45 & 6.92 & 7.39 & 6.76 & 7.47 & 7.57 & 7.42 & 7.18 & 7.37 \\
\hline $\mathrm{CaO}$ & 12.84 & 12.72 & 12.88 & 12.29 & 12.20 & 13.22 & 11.13 & 11.40 & 11.18 & 11.99 & 14.09 & 11.84 & 12.47 & 12.66 & 12.82 & 13.70 & 12.82 & 12.69 & 14.30 & 12.79 & 12.78 & 13.72 & 12.85 & 12.83 \\
\hline $\mathrm{Na}_{2} \mathrm{O}$ & 2.19 & 2.36 & 2.15 & 2.33 & 2.21 & 2.36 & 2.70 & 2.43 & 2.58 & 2.52 & 2.25 & 2.40 & 2.26 & 2.19 & 2.28 & 2.18 & 2.25 & 2.28 & 2.19 & 2.25 & 2.19 & 2.14 & 2.33 & 2.12 \\
\hline $\mathrm{K}_{2} \mathrm{O}$ & 0.06 & 0.05 & 0.04 & 0.10 & 0.08 & 0.12 & 0.18 & 0.08 & 0.45 & 0.18 & 0.13 & 0.10 & 0.08 & 0.06 & 0.07 & 0.10 & 0.07 & 0.08 & 0.09 & 0.09 & 0.09 & 0.09 & 0.06 & 0.05 \\
\hline $\mathrm{P}_{2} \mathrm{O}_{5}$ & 0.15 & 0.13 & 0.11 & 0.13 & 0.12 & 0.10 & 0.08 & 0.10 & 0.09 & 0.12 & 0.13 & 0.10 & 0.11 & 0.09 & 0.10 & 0.11 & 0.13 & 0.12 & 0.10 & 0.09 & 0.09 & 0.11 & 0.10 & 0.10 \\
\hline $\mathrm{CO}_{2}$ & 0.43 & 0.22 & 0.12 & 0.11 & 0.31 & 1.88 & 0.51 & 0.14 & 0.0 & 1.01 & 1.60 & 0.12 & 0.12 & 0.11 & 0.14 & 0.84 & 0.18 & 0.24 & 1.34 & 0.23 & 0.18 & 0.17 & 0.25 & 0.08 \\
\hline $\mathrm{H}_{2} \mathrm{O}^{+}$ & L.05 & 1.01 & 1.10 & 0.85 & 0.88 & 1.94 & 1.80 & 2.06 & 2.36 & 1.92 & 1.52 & 1.77 & 1.18 & 1.37 & 1.22 & 1.46 & 1.15 & 0.98 & 0.61 & 0.92 & 1.15 & 1.00 & 1.29 & 0.99 \\
\hline Sum & 100.21 & 99.90 & 99.86 & 100.59 & 99.88 & 100.13 & 100.47 & 99.16 & 99.12 & 99.12 & 100.03 & 99.77 & 99.11 & 100.32 & 99.91 & 99.75 & 100.87 & 100.05 & 100.35 & 99.71 & 100.46 & 100.91 & 100.33 & 99.81 \\
\hline $\mathrm{H}_{2} \mathrm{O}^{-}$ & 0.83 & 0.94 & 0.79 & 0.49 & 0.64 & 2.61 & 1.37 & 2.45 & 2.56 & 2.54 & 1.57 & 1.70 & 0.83 & 0.66 & 0.96 & 1.24 & 0.53 & 0.43 & 0.53 & 0.76 & 0.82 & 0.43 & 0.84 & 0.84 \\
\hline Féo Total & 10.03 & 9.02 & 9.81 & 10.25 & 9.66 & 8.34 & 8.24 & 8.16 & 7.36 & 7.29 & 7.94 & 8.56 & 8.98 & 9.23 & 8.81 & 8.84 & 8.99 & 8.99 & 8.36 & 8.79 & 9.00 & 8.79 & 8.46 & 9.21 \\
\hline Mg No. & 56.40 & 59.25 & 57.53 & 57.34 & 60.23 & 61.53 & 62.76 & 63.86 & 66.43 & 65.89 & 63.17 & 62.59 & 62.63 & 61.67 & 61.47 & 59.60 & 60.90 & 62.44 & 62.06 & 63.22 & 62.98 & 63.07 & 63.20 & 61.83 \\
\hline
\end{tabular}

TABLE 1A - Continued

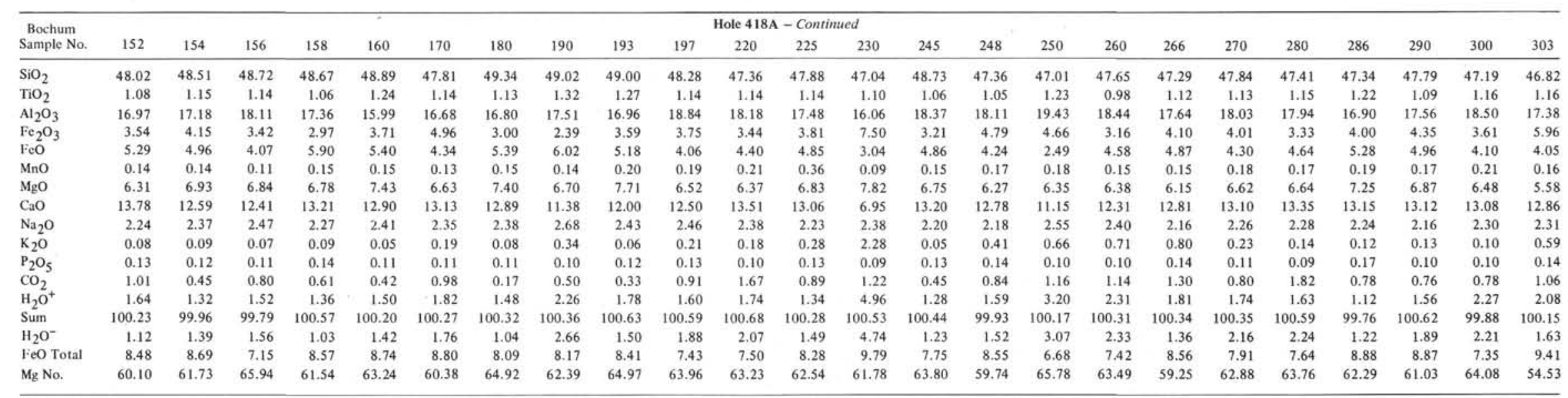




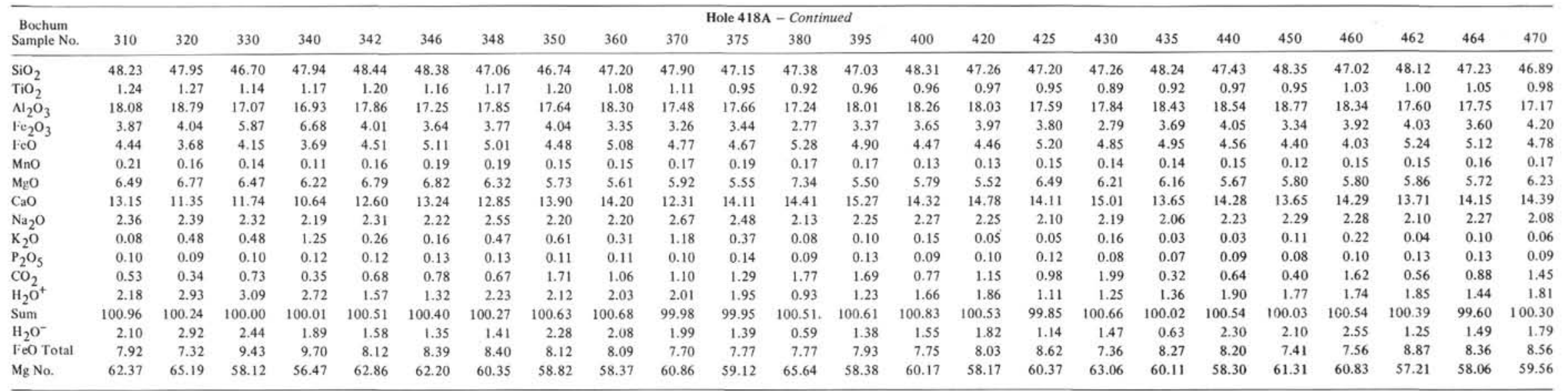

TABLE 1A - Continued

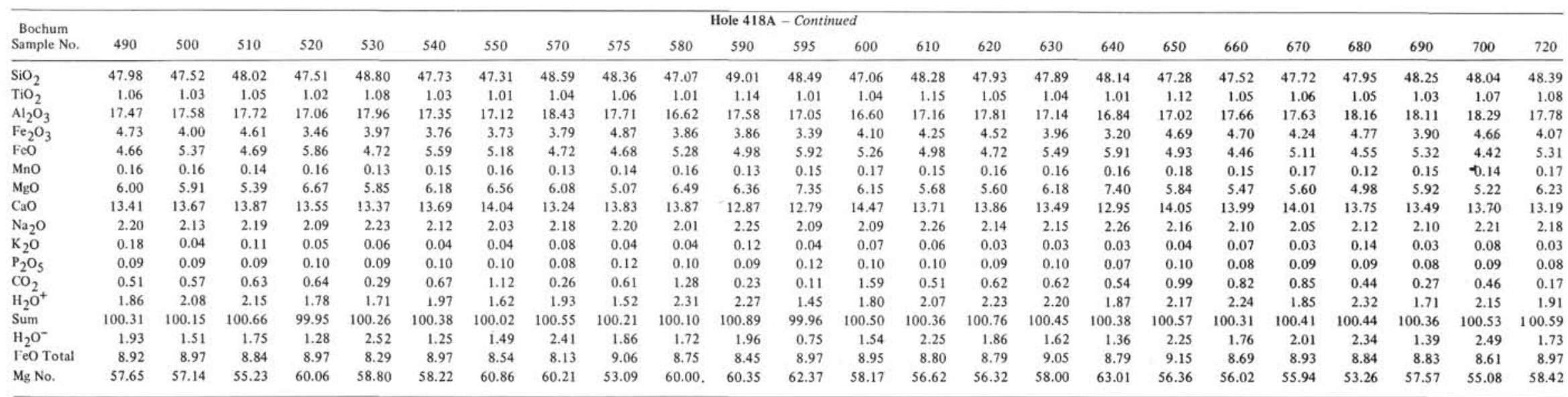


TABLE 1A - Continued

\begin{tabular}{|c|c|c|c|c|c|c|c|c|c|c|c|c|c|c|c|c|c|c|c|c|c|c|c|c|}
\hline \multirow{2}{*}{$\begin{array}{c}\begin{array}{c}\text { Bochum } \\
\text { Sample No. }\end{array} \\
\text {. }\end{array}$} & \multirow[b]{2}{*}{730} & \multirow[b]{2}{*}{740} & \multirow[b]{2}{*}{750} & \multirow[b]{2}{*}{760} & \multirow[b]{2}{*}{770} & \multirow[b]{2}{*}{780} & \multirow[b]{2}{*}{790} & \multirow[b]{2}{*}{800} & \multirow[b]{2}{*}{810} & \multicolumn{4}{|c|}{ Hole $418 \mathrm{~A}$ - Continued } & \multirow[b]{2}{*}{860} & \multirow[b]{2}{*}{870} & \multirow[b]{2}{*}{880} & \multirow[b]{2}{*}{890} & \multirow[b]{2}{*}{900} & \multirow[b]{2}{*}{910} & \multirow[b]{2}{*}{920} & \multirow[b]{2}{*}{940} & \multirow[b]{2}{*}{950} & \multirow[b]{2}{*}{960} & \multirow[b]{2}{*}{970} \\
\hline & & & & & & & & & & 820 & 830 & 840 & 850 & & & & & & & & & & & \\
\hline $\mathrm{SiO}_{2}$ & 48.38 & 48.38 & 49.14 & 48.14 & 47.49 & 48.29 & 48.08 & 48.48 & 47.86 & 47.14 & 48.66 & 48.19 & 47.75 & 47.38 & 47.70 & 49.04 & 47.73 & 47.66 & 47.73 & 47.53 & 48.14 & 47.79 & 47.88 & 48.16 \\
\hline $\mathrm{TiO}_{2}^{2}$ & 1.13 & 0.95 & 1.31 & 1.28 & 1.24 & 1.20 & 1.21 & 1.42 & 1.58 & 1.49 & 1.54 & 1.57 & 1.34 & 1.35 & 1.27 & 1.31 & 1.25 & 1.31 & 1.29 & 1.31 & 1.22 & 1.24 & 1.32 & 1.27 \\
\hline $\mathrm{Al}_{2} \mathrm{O}_{3}$ & 16.77 & 18.33 & 15.60 & 15.18 & 16.81 & 16.44 & 16.39 & 17.01 & 16.51 & 15.94 & 15.37 & 15.58 & 17.46 & 17.34 & 16.48 & 16.79 & 16.70 & 17.47 & 16.98 & 16.21 & 16.31 & 15.92 & 16.08 & 15.71 \\
\hline $\mathrm{Fe}_{2} \mathrm{O}_{3}$ & 3.62 & 3.78 & 3.27 & 3.25 & 3.18 & 3.83 & 3.25 & 3.78 & 4.95 & 4.92 & 4.41 & 4.76 & 3.37 & 4.25 & 4.01 & 4.19 & 5.01 & 4.15 & 4.03 & 3.76 & 3.36 & 3.71 & 3.30 & 2.68 \\
\hline $\mathrm{FeO}$ & 5.44 & 5.06 & 6.62 & 6.62 & 5.62 & 5.75 & 5.93 & 6.14 & 5.51 & 5.27 & 6.55 & 6.44 & 5.78 & 5.22 & 5.33 & 5.24 & 4.74 & 4.14 & 5.02 & 5.71 & 6.29 & 5.91 & 5.83 & 7.09 \\
\hline MnO & 0.15 & 0.15 & 0.19 & 0.19 & 0.17 & 0.16 & 0.18 & 0.17 & 0.18 & 0.16 & 0.18 & 0.18 & 0.16 & 0.16 & 0.16 & 0.13 & 0.15 & 0.14 & 0.15 & 0.19 & 0.18 & 0.17 & 0.16 & 0.18 \\
\hline $\mathrm{MgO}$ & 6.49 & 6.33 & 7.48 & 7.52 & 6.85 & 6.67 & 7.00 & 6.28 & 6.53 & 6.95 & 6.38 & 6.56 & 6.04 & 5.76 & 6.05 & 6.24 & 5.70 & 6.56 & 6.50 & 6.94 & 7.03 & 6.70 & 6.71 & 7.06 \\
\hline $\mathrm{CaO}$ & 13.07 & 13.42 & 12.49 & 13.41 & 14.02 & 13.60 & 13.62 & 12.88 & 12.35 & 12.67 & 11.91 & 11.83 & 13.50 & 13.20 & 14.10 & 12.31 & 13.75 & 13.04 & 13.15 & 13.34 & 13.64 & 13.91 & 13.60 & 13.70 \\
\hline $\mathrm{Na}_{2} \mathrm{O}$ & 2.41 & 2.10 & 2.31 & 2.24 & 2.31 & 2.18 & 2.20 & 2.30 & 2.36 & 2.22 & 2.33 & 2.45 & 2.43 & 2.35 & 2.33 & 2.55 & 2.36 & 2.49 & 2.52 & 2.28 & 2.24 & 2.20 & 2.28 & 2.22 \\
\hline $\mathrm{K}_{2} \mathrm{O}$ & 0.25 & 0.02 & 0.05 & 0.06 & 0.05 & 0.03 & 0.04 & 0.09 & 0.04 & 0.04 & 0.08 & 0.30 & 0.13 & 0.26 & 0.09 & 0.31 & 0.34 & 0.04 & 0.04 & 0.05 & 0.04 & 0.05 & 0.09 & 0.06 \\
\hline $\mathrm{P}_{2} \mathrm{O}_{5}$ & 0.09 & 0.09 & 0.11 & 0.13 & 0.14 & 0.13 & 0.12 & 0.13 & 0.16 & 0.14 & 0.1 & 0.14 & 0.13 & 0.13 & 0.13 & 0.12 & 0.13 & 0.13 & 0.14 & 0.13 & 0.13 & 0.14 & 0.15 & 0.13 \\
\hline $\mathrm{CO}_{2}$ & 1.43 & 0.17 & 0.32 & 1.06 & 1.25 & 0.81 & 0.89 & 0.37 & 0.38 & 1.13 & 0.19 & 0.40 & 1.15 & 1.08 & 0.98 & 0.18 & 1.00 & 1.14 & 0.76 & 0.95 & 0.92 & 1.34 & 1.44 & 1.12 \\
\hline $\mathrm{H}_{2} \mathrm{O}^{+}$ & 1.44 & 1.52 & 1.19 & 0.80 & 1.21 & 1.52 & 0.89 & 1.32 & 1.90 & 1.78 & 1.37 & 1.16 & 1.03 & 1.31 & 1.24 & 1.87 & 1.60 & 2.20 & 1.81 & 1.49 & 1.09 & 1.29 & 1.35 & 0.87 \\
\hline Sum & 100.67 & 100.30 & 100.08 & 99.88 & 100.34 & 100.61 & 99.80 & 100.37 & 100.31 & 99.85 & 99.11 & 99.56 & 100.27 & 99.79 & 99.87 & 100.28 & 100.46 & 100.47 & 100.12 & 99.89 & 100.59 & 100.37 & 100.19 & 100.25 \\
\hline $\mathrm{H}_{2} \mathrm{O}^{-}$ & 2.29 & 1.67 & 1.26 & 0.89 & 1.00 & 1.25 & 0.98 & 1.32 & 1.90 & 2.17 & 0.77 & 1.22 & 1.21 & 1.38 & 1.44 & 1.92 & 1.52 & 2.52 & 2.26 & 1.68 & 1.10 & 0.77 & 1.36 & 0.62 \\
\hline FeO Tot: & 8.70 & 8.46 & 9.56 & 9.54 & 8.48 & 9.20 & 8.85 & 9.54 & 9. & 9. & 10.52 & 10.72 & 8.81 & 9.04 & 8.94 & 9.01 & 9.25 & 7.87 & 8.65 & 9.09 & 9.31 & 9.25 & 8.80 & 9.50 \\
\hline Mg No. & 60.16 & 60.22 & 61.28 & 61.45 & 62.04 & 59.47 & 61.53 & 57.11 & 57.01 & 59.18 & 55.10 & 55.31 & 58.10 & 56.30 & 57.80 & 58.35 & 55.50 & 62.76 & 60.33 & 60.69 & 60.43 & 59.44 & 60.67 & 60.05 \\
\hline
\end{tabular}

TABLE 1A - Continued

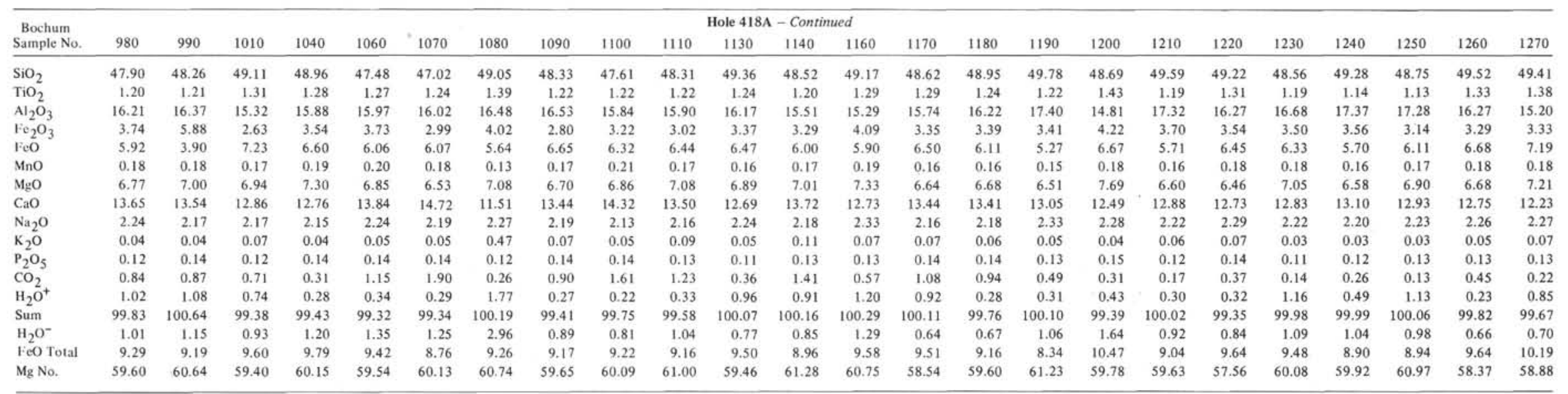


TABLE 1B

Trace-Element Analyses of Whole-Rock Samples From DSDP Hole 418A

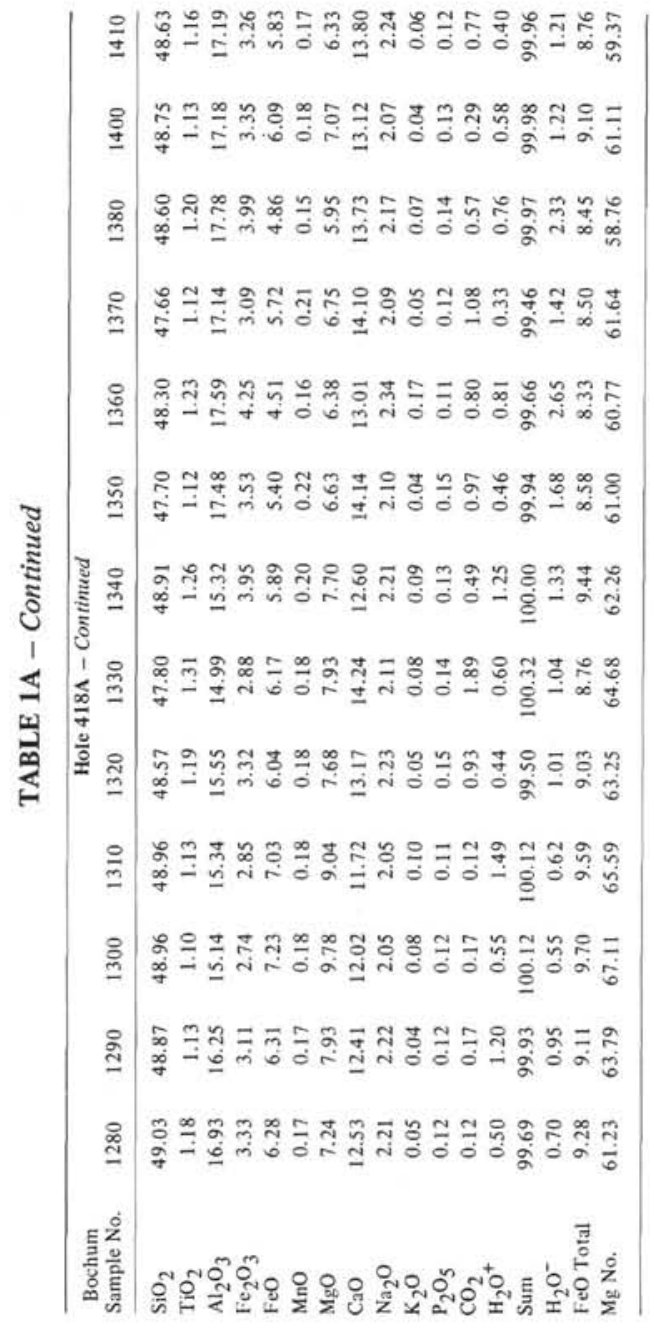

\begin{tabular}{|c|c|c|c|c|c|c|}
\hline $\begin{array}{l}\text { Bedford } \\
\text { College } \\
\text { XRF Numbers }\end{array}$ & $\mathrm{Nb}$ & $Y$ & $\begin{array}{l}\mathrm{Sr} \\
(\mathrm{ppm})\end{array}$ & $\mathrm{Rb}$ & $\mathrm{Zr}$ & $\begin{array}{l}\text { Bochum } \\
\text { Code No. }\end{array}$ \\
\hline 865901 & 4 & 24 & 120 & 0 & 56 & 40 \\
\hline 867801 & 4 & 25 & 105 & 1 & 56 & 60 \\
\hline 864701 & 5 & 23 & 115 & 0 & 54 & 90 \\
\hline 866801 & 5 & 21 & 101 & 0 & 55 & 120 \\
\hline 863701 & 4 & 21 & 108 & 2 & 49 & 130 \\
\hline 866701 & 4 & 24 & 106 & 2 & 43 & 140 \\
\hline 866481 & 5 & 23 & 106 & 0 & 64 & 150 \\
\hline 868101 & 7 & 23 & 104 & 1 & 62 & 290 \\
\hline 864901 & 4 & 25 & 116 & 0 & 52 & 300 \\
\hline 864201 & 5 & 25 & 112 & 2 & 61 & 310 \\
\hline 865701 & 4 & 25 & 152 & 0 & 45 & 360 \\
\hline 865001 & 5 & 21 & 106 & 5 & 45 & 460 \\
\hline 863101 & 4 & 25 & 121 & 2 & 48 & 470 \\
\hline 864601 & 5 & 24 & 100 & 0 & 49 & 480 \\
\hline 864101 & 5 & 23 & 101 & 0 & 44 & 500 \\
\hline 865301 & 5 & 27 & 100 & 1 & 62 & 510 \\
\hline 866901 & 5 & 22 & 98 & 0 & 48 & 520 \\
\hline 866601 & 4 & 21 & 99 & 2 & 43 & 540 \\
\hline 864001 & 4 & 23 & 106 & 0 & 50 & 550 \\
\hline 864301 & 2 & 21 & 105 & 1 & 42 & 580 \\
\hline 864501 & 4 & 25 & 110 & 0 & 49 & 600 \\
\hline 866501 & 4 & 24 & 94 & 0 & 53 & 610 \\
\hline 868001 & 2 & 24 & 105 & 0 & 52 & 620 \\
\hline 865201 & 5 & 23 & 101 & 1 & 51 & 660 \\
\hline 867901 & 5 & 25 & 114 & 0 & 51 & 670 \\
\hline 863501 & 4 & 22 & 95 & 0 & 44 & 680 \\
\hline 865501 & 4 & 23 & 100 & 0 & 48 & 740 \\
\hline 861501 & 4 & 27 & 115 & 0 & 63 & 770 \\
\hline 861901 & 5 & 26 & 112 & 0 & 62 & 790 \\
\hline 862401 & 4 & 29 & 100 & 0 & 77 & 800 \\
\hline 867601 & 6 & 31 & 114 & 1 & 85 & 810 \\
\hline 870701 & 6 & 30 & 112 & 0 & 78 & 820 \\
\hline 863901 & 7 & 32 & 104 & 0 & 74 & 830 \\
\hline 865801 & 5 & 28 & 108 & 1 & 69 & 860 \\
\hline 863001 & 6 & 28 & 108 & 1 & 66 & 870 \\
\hline 861801 & 4 & 26 & 99 & 9 & 60 & 890 \\
\hline 866201 & 5 & 25 & 106 & 0 & 61 & 910 \\
\hline 865101 & 5 & 29 & 108 & 0 & 66 & 920 \\
\hline 861601 & 2 & 28 & 103 & 0 & 59 & 940 \\
\hline 866101 & 5 & 23 & 114 & 0 & 65 & 950 \\
\hline 867501 & 5 & 26 & 113 & 1 & 63 & 960 \\
\hline 862501 & 4 & 26 & 108 & 0 & 71 & 980 \\
\hline 862801 & 2 & 26 & 109 & 1 & 62 & 990 \\
\hline 862301 & 7 & 27 & 132 & 3 & 65 & 1010 \\
\hline 867101 & 4 & 25 & 100 & 0 & 67 & 1040 \\
\hline 867701 & 6 & 27 & 103 & 0 & 66 & 1060 \\
\hline $8664 \mathrm{Al}$ & 5 & 26 & 114 & 0 & 67 & 1070 \\
\hline 863801 & 2 & 26 & 110 & 1 & 59 & 1100 \\
\hline 862201 & 7 & 27 & 105 & 1 & 74 & 1110 \\
\hline 862601 & 4 & 24 & 108 & 4 & 68 & 1130 \\
\hline 867301 & 2 & 25 & 111 & 2 & 61 & 1140 \\
\hline 866001 & 2 & 26 & 111 & 2 & 66 & 1150 \\
\hline 863401 & 7 & 28 & 108 & 1 & 66 & 1160 \\
\hline 868201 & 4 & 28 & 108 & 0 & 67 & 1170 \\
\hline 865401 & 6 & 28 & 106 & 0 & 63 & 1180 \\
\hline 870801 & 2 & 26 & 99 & 0 & 52 & 1210 \\
\hline 862101 & 6 & 28 & 100 & 1 & 65 & 1220 \\
\hline 863601 & 5 & 24 & 98 & 2 & 49 & 1250 \\
\hline 863301 & 5 & 28 & 98 & 0 & 70 & 1260 \\
\hline 866301 & 4 & 30 & 97 & 0 & 69 & 1270 \\
\hline 862701 & 5 & 26 & 97 & 0 & 59 & 1280 \\
\hline 865601 & 4 & 26 & 98 & 0 & 58 & 1290 \\
\hline 861701 & 6 & 27 & 87 & 0 & 58 & 1300 \\
\hline 867401 & 5 & 25 & 87 & 2 & 63 & 1310 \\
\hline 864801 & 4 & 28 & 103 & 0 & 57 & 1320 \\
\hline 867001 & 5 & 22 & 130 & 4 & 59 & 1330 \\
\hline 864401 & 5 & 28 & 103 & 0 & 63 & 1340 \\
\hline 863201 & 5 & 23 & 102 & 0 & 70 & 1350 \\
\hline 862001 & 7 & 22 & 103 & 0 & 53 & 1370 \\
\hline 862901 & 5 & 27 & 97 & 0 & 54 & 1400 \\
\hline 867201 & 4 & 26 & 102 & 0 & 60 & 1410 \\
\hline \multicolumn{7}{|l|}{ Standards } \\
\hline 108801 & 96 & 26 & 1288 & 46 & 248 & BR \\
\hline 110601 & 14 & 33 & 313 & 44 & 174 & BCR \\
\hline 110301 & 14 & 21 & 673 & 70 & 228 & AGV \\
\hline 256601 & 228 & 94 & 6 & 2014 & 758 & MICA-FE \\
\hline 110401 & 3 & 0 & 3 & 2 & 2 & PCC-1 \\
\hline 868401 & 61 & 22 & 727 & 76 & 254 & B-BO \\
\hline 868301 & 6 & 39 & 126 & 0 & 127 & AII \\
\hline 870601 & 16 & 25 & 72 & 361 & 187 & G-BO \\
\hline
\end{tabular}


and in the pillow basalts of Unit 4. They are usually filled with carbonate accompanied by brown or green smectite.

\section{Hole 418A}

The basement section at Site 418 is similar to that at Site 417. Sixteen lithologic units were identified in Hole 418A, comprising pillowed and massive flows, various breccias, and a series of dikes (lithologic Unit 15) at the base of the section. Most basalts are moderately to sparsely phyric, aphyric varieties occurring only rarely in Units $14 \mathrm{~A}$ through C. Phenocrysts are mostly of plagioclase with lesser amounts of clinopyroxene, olivine, spinel, and possibly some iron-titanium oxides. Plagioclase occurs as euhedral to rounded crystals and forms up to 20 modal per cent. Compositions range from $\mathrm{An}_{80}$ to $\mathrm{An}_{60}$ with both normal and oscillatory zoning. Glass inclusions are common and occur irregularly distributed or in zonal arrangement. Bubbles within these inclusions suggest the presence of a fluid phase at the time of crystallization. Except for lithologic Unit 9, all the basalts contain 3 to 5 modal per cent of olivine phenocrysts, most of which is replaced by brown smectite and/or carbonate. Olivine pseudomorphs are euhedral to rounded. In lithologic Units 6 and 8 rounded crystals of fresh olivine coexist with pseudomorphs, suggesting two generations of olivine crystallization. Small amounts of phenocrystic clinopyroxene occur in lithologic Units 6 through 15 . These crystals are fresh, subhedral to anhedral or rounded, and typically form glomerocrysts with olivine and plagioclase. Euhedral spinel is a common phase in lithologic Unit 6. It is dark red-brown and often rimmed by magnetite. The spinel occurs both as single grains and as inclusions in plagioclase and olivine.

Groundmass textures vary from fine-grained quenching pillow basalts to medium-grained subophitic or "ophimottled" in massive flows. Skeletal crystals of plagioclase, clinopyroxene, olivine (altered to smectite), iron-titanium oxides, and sulfides are the main primary matrix phases. Glass is always present but is usually devitrified or altered. Vesicles and amygdules are variable in distribution and size, and most are filled with brown smectite and carbonate. In addition, plagioclase may be altered to light brown smectite.

The six dikes that cut lithologic Unit 14 can be subdivided into the following two groups: the first with the phenocryst assemblage plagioclase, olivine, and clinopyroxene, the second with plagioclase and olivine only as phenocrysts.

\section{BASALT CHEMISTRY}

Following previous compositional studies of drilled oceanic basalt (e.g., Flower et al., 1977; 1978; Byerly and Wright, in press), we have normalized major element analyses to a $\mathrm{H}_{2} \mathrm{O}^{+}$-free basis with total iron oxide expressed as $\mathrm{FeO}$, assuming all water to be post-magmatic. A correction for added carbonate (mostly as $\mathrm{CaCO}_{3}$ in veins and vugs) was made prior to normalization by subtracting a $\mathrm{CO}_{2}$-equivalent $\mathrm{CaO}$ component from each analysis. Some corrected analyses show the effects of $\mathrm{Ca}^{+2}$ and $\mathrm{Mg}^{+2}$ leaching and enrichment in $\mathrm{SiO}_{2}$ and $\mathrm{K}_{2} \mathrm{O}$. These appear to be the main changes in major element composition associated with smectite growth during sea-water percolation through the basalt pile. Empirical studies of fresh altered sample pairs from single cooling units (Flower et al., this volume) indicate that for both $\mathrm{H}_{2} \mathrm{O}^{+}$and $\mathrm{K}_{2} \mathrm{O}<0.20$ wt. per cent, leaching and addition effects are virtually negligible (except for $\mathrm{K}_{2} \mathrm{O}$ and some trace elements). Hence, carbonate-corrected normalized analyses probably closely approximate original magmatic compositions.

Whole-rock compositional data for Holes 417D and 418A (Tables $3 \mathrm{~A}$ and $\mathrm{B}$ ) are used to define oxide variations, to make deductions about magmatic fractionation processes, and, by reference to lithologic and magnetic data, to refine stratigraphic divisions in the core. These divisions are further evaluated in light of the chemical alteration profiles at each drill site.

\section{Stratigraphic Evaluation}

\section{Hole 417A}

Shipboard analyses for Hole 417A reflect extreme alteration of the basalts (see Donnelly et al., this volume) with $\mathrm{K}_{2} \mathrm{O}$ contents up to 6 wt. per cent and $\mathrm{CaO}$ contents less than 5 wt. per cent. Fresh material is rare or absent. The eight analyses listed in Table 1 were selected as the freshest available. These samples are from the lower part of the section (Cores 33 to 44 ) and suggest that original magma compositions in Hole 417A were indistinguishable from those in Hole 417D. However, the overall intensity of alteration in Hole 417A precludes detailed stratigraphic evaluation of magma chemistry. Magnetic stratigraphy is simple, with stable inclinations close to the predicted dipole.

\section{Hole 417D}

At Hole 417D, samples from each lithologic unit were analyzed. Except for the uppermost 2 to 3 meters of basement, alteration is slight and is concentrated along eruptive unit boundaries and in breccia zones. Stratigraphic boundaries of the eight glass compositional groups identified by Byerly and Sinton (this volume) are matched by changes in whole-rock composition (Figure 2). Chemical breaks illustrated by parameters such as $\mathrm{TiO}_{2} / \mathrm{Al}_{2} \mathrm{O}_{3}$ ratios (reflecting fractionation of plagioclase) and $\mathrm{Mg} /\left(\mathrm{Mg}+\mathrm{Fe}^{+2}\right)$ ratios (reflecting fractionation of mafic phases) also commonly correlate with lithologic and stable magnetic unit boundaries (Figure 2). Because of this coincidence of chemical compositions with other parameters, the chemical data have been used to define an eruptive stratigraphy.

Theoretically, the products of a single eruption sampled by drilling should have: (1) uniform glass compositions, (2) whole-rock compositional variability consistent with effects of post-eruption fractionation, (3) uniform magnetic polarities and stable inclinations, (4) lithology consistent with the chemical data, and (5) no major sedimentary or clastic intercalations. In practice, however, the chemical, lithologic, and magnetic character of single eruptive units can undergo considerable variation. Variations in glass compositions can arise from nonhomogeneities in the sub-rift magma chamber, and whole-rock compositions can reflect extensive phenocryst redistribution and post-eruptive crystallization. Stable magnetic inclinations can reflect differential subsidence and rotation of crustal blocks during a single eruption, and lithologies can vary markedly as a result of 
TABLE 2

DSDP Numbers Corresponding to Those of the Bochum Laboratory and Computer File for Samples Analyzed

\begin{tabular}{ccc}
\hline Bochum Number & $\begin{array}{c}\text { DSDP Sample Number } \\
\text { (Interval in cm) }\end{array}$ & Piece Number \\
\hline Hole 417A & & \\
110 & $33-5,54-58$ & $5 \mathrm{~A}$ \\
120 & $34-2,88-93$ & $8 \mathrm{~A}$ \\
160 & $37-1,37-40$ & $1 \mathrm{~B}$ \\
180 & $38-3,75-79$ & 10 \\
210 & $40-3,25-29$ & 1 \\
220 & $41-3,92-97$ & 2 \\
240 & $44-2,13-17$ & $1 \mathrm{~A}$ \\
250 & $44-3,58-68$ & 9
\end{tabular}

Hole 417D

1020

1040

1060

1080

1110

1120

1140

1150

1170

1180

1185

1210

1240

1275

1280

1300

1320

1330

1400

1440

1460

1480

1500

1510

1520

1530

1540

1550

1560

1570

1580

1590

1600

1620

1630

1640

1650

1660

1670

1690

1710

1720

1730

1740

1750

1760

1780

1790

Hole 418A

$\begin{array}{lll} & & \\ 10 & 15 \mathrm{~A} & \\ 15 & 15-1,27-29 & 2 \mathrm{~A} \\ 20 & 15-1,52-56 & 1 \mathrm{~L} \\ 30 & 16-1,72-74 & 5 \mathrm{~B} \\ \end{array}$

$22-4, \overline{24}-28$

22-6, $143-148 \quad 8$

$\begin{array}{ll}26-6,87-91 & 3 \mathrm{~B} \\ 28-1,44-50 & 4 \mathrm{~A}\end{array}$

28-6, 111-117 4

29-6, 8-13

$30-2,82-86 \quad 2 \mathrm{~A}$

$30-7,90-96 \quad 3$

$\begin{array}{ll}31-2,98-102 & 2 \mathrm{~F} \\ 31-4,78-80 & 3 \mathrm{~A}\end{array}$

$33-4,47-52 \quad 3 \mathrm{~B}$

35-4, 27-33

$36-3,127-129$

$36-4,36-39$

$38-4,106-111$

39-3, 95-101

$42-1,10-16$

$43-3,52-57$

$44-1,54-59$

45-1, 5-8

48-6, 113-115

49-1, 109-111

49-3, 55-57

$50-2,73-75$

$52-3,87-89$

$52-5,20-22$

53-2, 109-111

54-1, 89-91

54-6, 21-23

55-2, 7-9

$55-4,110-112$

$57-2,62-64$

$57-3,44-46$

58-1, 127-129

$59-1,121-123$

59-5, 82-84

60-3, 109-111

62-4, 36-38

63-3, 105-107

64-1, 53-55

65-1, 111-113

65-5, 23-25

67-1, 56-58

$67-2,71-73$

68-5, 83-85

69-2, 9-11
$1 \mathrm{~A}$

(A)

4

$1 \mathrm{~F}$

2

6

1B

1

7B

$4 \mathrm{~B}$

10

$6 \mathrm{~B}$

4
10

2

10

$1 \mathrm{~F}$

10

1

A

A

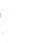

A

A

A

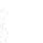

D

C

B

.

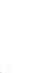

B

B

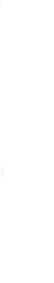

TABLE 2 - Continued

\begin{tabular}{|c|c|c|}
\hline Bochum Number & $\begin{array}{l}\text { DSDP Sample Number } \\
\text { (Interval in } \mathrm{cm} \text { ) }\end{array}$ & Piece Number \\
\hline 40 & $17-1,134-139$ & $3 \mathrm{C}$ \\
\hline 50 & $17-3,124-126$ & 11 \\
\hline 55 & $18-1,66-68$ & $2 \mathrm{E}$ \\
\hline 60 & $18-2,33-38$ & $2 \mathrm{~A}$ \\
\hline 70 & $18-2,99-123$ & $3 \mathrm{C}$ \\
\hline 80 & $18-4,26-29$ & $1 \mathrm{~A}$ \\
\hline 90 & $18-6,89-94$ & 3B \\
\hline 100 & $19-2,57-61$ & $1 \mathrm{C}$ \\
\hline 103 & $19-3,66-68$ & 1D \\
\hline 106 & $19-6,100-102$ & $3 \mathrm{~F}$ \\
\hline 110 & $20-1,103-108$ & $6 \mathrm{~F}$ \\
\hline 120 & $20-2,68-73$ & $1 \mathrm{G}$ \\
\hline 130 & $20-3,49-54$ & $1 \mathrm{G}$ \\
\hline 133 & $20-4,7-9$ & $1 \mathrm{~A}$ \\
\hline 136 & $20-5,40-42$ & $2 \mathrm{~A}$ \\
\hline 140 & $20-7,101-104$ & 3 \\
\hline 146 & $22-1,129-131$ & $11 \mathrm{~B}$ \\
\hline 150 & $22-2,55-59$ & $1 \mathrm{G}$ \\
\hline 152 & $23-1,22-26$ & 2 \\
\hline 154 & $24-1,103-105$ & $3 \mathrm{~B}$ \\
\hline 156 & $24-2,71-74$ & 4 \\
\hline 158 & $25-1,36-38$ & 2 \\
\hline 160 & $25-2,145-148$ & $5 \mathrm{G}$ \\
\hline 170 & $26-1,16-20$ & $1 \mathrm{~A}$ \\
\hline 180 & $26-2,76-80$ & 2D \\
\hline 190 & $26-4,144-147$ & - \\
\hline 193 & $27-1,24-26$ & - \\
\hline 195 & $27-1,133-135$ & - \\
\hline 197 & $27-2,83-85$ & - \\
\hline 215 & $28-2,130-132$ & 10B \\
\hline 220 & $28-4,20-24$ & $1 \mathrm{~B}$ \\
\hline 225 & $28-5,11-13$ & 1 \\
\hline 230 & $29-1,97-100$ & 8 \\
\hline 245 & $30-2,124-126$ & $6 \mathrm{~B}$ \\
\hline 248 & $31-1,98-100$ & 6 \\
\hline 250 & $31-3,103-106$ & 11 \\
\hline 260 & $33-1,75-78$ & - \\
\hline 266 & $33-2,85-87$ & $6 \mathrm{~A}$ \\
\hline 270 & $33-4,105-108$ & $6 \mathrm{~B}$ \\
\hline 280 & $33-4,136-139$ & $6 \mathrm{E}$ \\
\hline 286 & $33-6,59-61$ & $5 \mathrm{~A}$ \\
\hline 290 & $34-1,89-93$ & $2 \mathrm{~F}$ \\
\hline 292 & $34-4,15-17$ & $1 \mathrm{~B}$ \\
\hline 294 & $35-1,21-23$ & $3 \mathrm{~A}$ \\
\hline 300 & $35-4,93-99$ & $3 \mathrm{~F}$ \\
\hline 303 & $36-1,100-103$ & $8 \mathrm{C}$ \\
\hline 310 & $36-3,80-84$ & $3 \mathrm{D}$ \\
\hline 320 & $36-5,136-139$ & - \\
\hline 330 & $38-1,75-78$ & $3 B$ \\
\hline 340 & $38-3,128-130$ & 7 \\
\hline 342 & $38-4,77-79$ & $5 \mathrm{~A}$ \\
\hline 346 & $39-2,13-15$ & 1 \\
\hline 348 & $40-2,59-61$ & $2 \mathrm{D}$ \\
\hline 350 & $40-2,85-88$ & $3 \mathrm{~A}$ \\
\hline 355 & $41-1,32-34$ & $4 \mathrm{~A}$ \\
\hline 360 & $41-1,100-104$ & 7 \\
\hline 370 & $42-1,52-54$ & $2 \mathrm{E}$ \\
\hline 375 & $42-2,81-83$ & 6 \\
\hline 380 & $42-3,11-15$ & $1 \mathrm{~A}$ \\
\hline 395 & $42-3,51-53$ & 3 \\
\hline 400 & $42-4,9-12$ & $1 \mathrm{~A}$ \\
\hline 420 & $42-5,27-30$ & 2 \\
\hline 425 & $42-5,65-67$ & 5 \\
\hline 430 & $43-1,78-80$ & $4 \mathrm{D}$ \\
\hline 435 & $43-1,109-111$ & $8 \mathrm{D}$ \\
\hline 440 & $43-1,136-140$ & $5 \mathrm{~B}$ \\
\hline 450 & $44-3,29-32$ & 3 \\
\hline 460 & $44-3,104-106$ & $6 \mathrm{~B}$ \\
\hline 462 & $44-5,42-44$ & $3 \mathrm{~A}$ \\
\hline
\end{tabular}

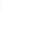

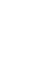

(1)


TABLE 2 - Continued

\begin{tabular}{|c|c|c|}
\hline Bochum Number & $\begin{array}{l}\text { DSDP Sample Number } \\
\text { (Interval in } \mathrm{cm} \text { ) }\end{array}$ & Piece Number \\
\hline 464 & $45-1,73-75$ & $5 \mathrm{C}$ \\
\hline 470 & $45-3,22-26$ & 1B \\
\hline 490 & $46-1,25-30$ & $2 \mathrm{~A}$ \\
\hline 500 & $46-2,77-81$ & $4 \mathrm{~A}$ \\
\hline 510 & $46-3,126-134$ & $8 \mathrm{~B}$ \\
\hline 520 & $46-4,31-36$ & $3 \mathrm{~B}$ \\
\hline 530 & $47-2,93-98$ & $4 \mathrm{~A}$ \\
\hline 540 & $47-2,123-126$ & $2 \mathrm{D}$ \\
\hline 550 & $47-5,102-105$ & 5 \\
\hline 570 & $48-1,136-142$ & $5 \mathrm{G}$ \\
\hline 575 & $48-3,50-52$ & $3 \mathrm{~A}$ \\
\hline 580 & $48-3,73-78$ & $3 \mathrm{G}$ \\
\hline 590 & $48-4,21-24$ & $2 \mathrm{G}$ \\
\hline 595 & $48-0,70-72$ & 2 \\
\hline 600 & $49-1,61-65$ & $3 \mathrm{G}$ \\
\hline 610 & $49-1,135-139$ & $6 \mathrm{E}$ \\
\hline 620 & $49-2,27-30$ & $1 \mathrm{G}$ \\
\hline 630 & $50-2,49-53$ & $3 \mathrm{~A}$ \\
\hline 640 & $50-3,131-133$ & $2 \mathrm{H}$ \\
\hline 650 & $50-4,42-45$ & $4 \mathrm{~A}$ \\
\hline 660 & $50-5,47-50$ & $3 \mathrm{~A}$ \\
\hline 670 & $51-2,13-16$ & $1 \mathrm{~A}$ \\
\hline 680 & $51-3,122-126$ & $6 \mathrm{~B}$ \\
\hline 690 & $51-4,57-60$ & $3 \mathrm{G}$ \\
\hline 700 & $51-5,21-26$ & 2 \\
\hline 720 & $52-1,122-125$ & 10 \\
\hline 730 & $53-1,108-113$ & 9 \\
\hline 740 & $53-2,128-130$ & $6 \mathrm{~B}$ \\
\hline 750 & $55-3,125-128$ & 8 \\
\hline 760 & $55-4,142-145$ & 12 \\
\hline 770 & $56-1,116-119$ & $6 \mathrm{~B}$ \\
\hline 780 & $56-4,21-24$ & 1B \\
\hline 790 & $56-7,22-26$ & 2 \\
\hline 800 & $56-7,69-74$ & - \\
\hline 810 & $60-1,27-30$ & $1 \mathrm{G}$ \\
\hline 820 & $60-4,144-148$ & $1 \mathrm{M}$ \\
\hline 830 & $61-1,15-18$ & 1 \\
\hline 840 & $62-4,3-8$ & $1 \mathrm{~A}$ \\
\hline 850 & $63-2,103-109$ & $4 \mathrm{~A}$ \\
\hline 860 & $63-2,126-130$ & 4B \\
\hline 870 & $64-4,77-81$ & $2 \mathrm{E}$ \\
\hline 880 & $65-1,139-140$ & 8 \\
\hline 890 & $65-3,21-26$ & 2 \\
\hline 900 & $65-4,110-113$ & $5 \mathrm{D}$ \\
\hline 910 & $65-5,22-26$ & $1 \mathrm{G}$ \\
\hline 920 & $66-3,29-34$ & 2 \\
\hline 940 & $66-6,127-131$ & $8 \mathrm{~A}$ \\
\hline 950 & $68-1,44-48$ & $2 \mathrm{G}$ \\
\hline 960 & $68-1,65-70$ & $2 \mathrm{E}$ \\
\hline 970 & $69-1,134-140$ & $2 \mathrm{C}$ \\
\hline 980 & $69-6,24-28$ & $2 \mathrm{~A}$ \\
\hline 990 & $69-6,124-129$ & $4 \mathrm{~B}$ \\
\hline 1010 & $70-5,40-44$ & $2 \mathrm{G}$ \\
\hline 1040 & $71-2,23-26$ & 1D \\
\hline 1060 & $72-2,89-94$ & $3 G$ \\
\hline 1070 & $72-3,23-28$ & $1 \mathrm{~B}$ \\
\hline 1080 & $72-3,130-134$ & 5 \\
\hline 1090 & $73-3,2-9$ & 1 \\
\hline 1100 & $73-3,43-46$ & $3 \mathrm{~A}$ \\
\hline 1110 & $73-4,124-129$ & $2 \mathrm{G}$ \\
\hline 1130 & $74-2,48-120$ & $3 \mathrm{~A}$ \\
\hline 1140 & $74-3,120-126$ & 1 \\
\hline 1160 & $76-3,89-92$ & $1 \mathrm{G}$ \\
\hline 1170 & $77-4,133-136$ & $2 \mathrm{E}$ \\
\hline 1180 & $78-3,96-99$ & $2 \mathrm{D}$ \\
\hline 1190 & $79-3,42-118$ & 1I \\
\hline 1200 & $79-4,18-22$ & $1 \mathrm{C}$ \\
\hline 1210 & $79-4,140-143$ & $2 \mathrm{G}$ \\
\hline 1220 & $80-2,46-49$ & 2 \\
\hline
\end{tabular}

TABLE 2 - Continued

\begin{tabular}{cll}
\hline Bochum Number & $\begin{array}{c}\text { DSDP Sample Number } \\
\text { (Interval in cm) }\end{array}$ & Piece Number \\
\hline 1230 & $80-2,132-134$ & 7 \\
1240 & $80-3,48-52$ & 2 \\
1250 & $80-5,35-39$ & $1 \mathrm{C}$ \\
1260 & $80-6,47-120$ & $6 \mathrm{~B}$ \\
1270 & $82-1,35-37$ & - \\
1280 & $83-1,15-19$ & $1 \mathrm{~B}$ \\
1290 & $84-2,43-38$ & $1 \mathrm{C}$ \\
1300 & $85-1,14-18$ & 1 \\
1310 & $85-2,80-84$ & 4 \\
1320 & $85-6,29-32$ & $3 \mathrm{C}$ \\
1330 & $85-7,97-101$ & 5 \\
1340 & $86-1,2-6$ & $1 \mathrm{~A}$ \\
1350 & $86-2,33-37$ & 3 \\
1360 & $86-2,47-50$ & $4 \mathrm{~A}$ \\
1370 & $86-2,66-69$ & $4 \mathrm{~B}$ \\
1380 & $86-3,100-112$ & 7 \\
1400 & $86-4,38-42$ & $1 \mathrm{D}$ \\
1410 & $86-4,48-124$ & $7 \mathrm{~A}$ \\
\hline
\end{tabular}

different eruptive rates and variable rift topography. We believe that glass compositions and magnetic inclinations are least likely to vary significantly during a single eruption. Consequently, we have defined eruptive units as stratigraphic sequences bounded by both chemical and magnetic breaks and, accordingly, have identified a maximum of 25 and a minimum of 13 eruptive units for the section penetrated at Hole 417D. The number of eruptive units may be underestimated because of difficulty in interpretation of the breccias and the possibility that different eruptions are chemically and magnetically indistinguishable. Alternatively, the number of units may be overestimated if intraeruption variation is greater than we have assumed. The eruptive units vary in thickness from 1 meter to about 60 meters, but because sampling by a single drill hole is unrepresentative, these thicknesses probably do not reflect original eruptive volumes.

These eruptive units can be grouped into at least three major sequences bounded by major stratigraphic discontinuities (Figure 2). The discontinuities are believed to be major eruptive breaks as evidenced by marked changes in basalt composition and magnetic inclinations, and often by the presence of breccia zones and altered horizons.

\section{Hole 418A}

All 16 lithologic units in Hole 418A were sampled for analysis. As in Hole 417D, glass groupings (Byerly and Sinton, this volume) complement whole-rock compositions and, when combined with magnetic inclinations, permit a detailed breakdown of the eruptive record (Figure 3). Byerly and Sinton (this volume) infer 11 stratigraphic glass groups and define a separate group for the dikes traversing lithologic Unit $14 \mathrm{C}$. The glass groups correspond well with the lithologic and magnetic units (Figure 3), but can be further divided on the basis of whole-rock variations of $\mathrm{TiO}_{2} / \mathrm{Al}_{2} \mathrm{O}_{3}$ ratios and $\mathrm{Mg} /\left(\mathrm{Mg}+\mathrm{Fe}^{+2}\right)$ ratios. Using the same criteria as for Hole 417D, we infer a maximum of 54 and a minimum of 32 eruptive units for the Hole $418 \mathrm{~A}$ section, not including the dikes (Figure 3). Downhole magnetic and chemical discontinuities are more numerous and 


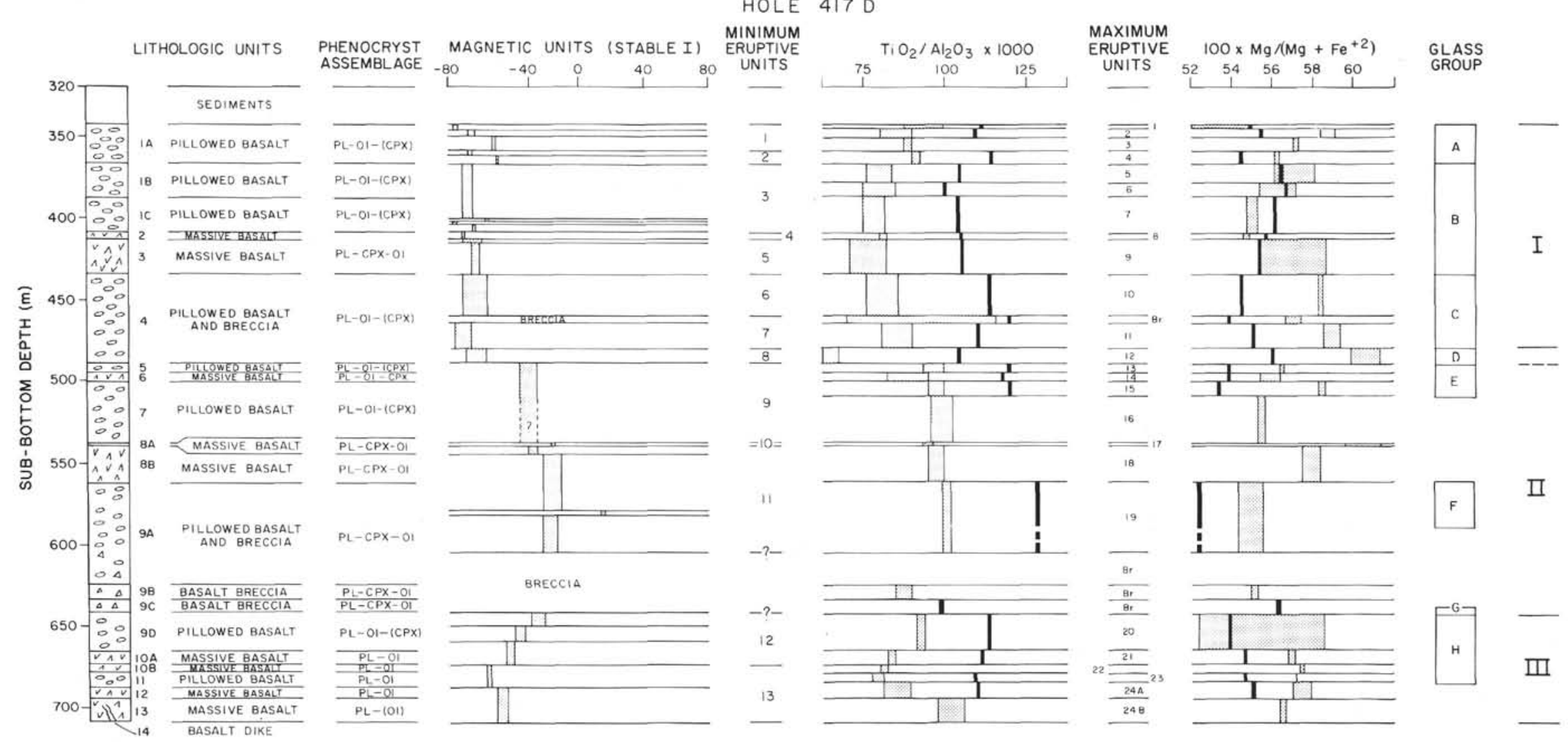

Figure 2. Hole 417D, summary of lithologic, magnetic, and chemical stratigraphy. Lithologic units are updated shipboard core divisions. Magnetic units are based on stable inclination variations measured onboard; the average Cretaceous dipole was $\pm 38^{\circ}$. Eruptive units are interpreted from downhole chemical and magnetic variations; average glass compositions (thick vertical lines) are shown in comparison to the range of whole-rock compositions for each eruptive unit in terms of $\mathrm{TiO}_{2} / \mathrm{Al}_{2} \mathrm{O}_{3}$ and $\mathrm{Mg} /\left(\mathrm{Mg}+\mathrm{Fe}^{+2}\right)$ ratios. Glass composition groups are after Byerly and Sinton (this volume): $\mathrm{BR}=$ breccia. $\mathrm{Note}: \mathrm{MgO}$ and FeO in eruptive Unit 1 reflect a high degree of alteration. 


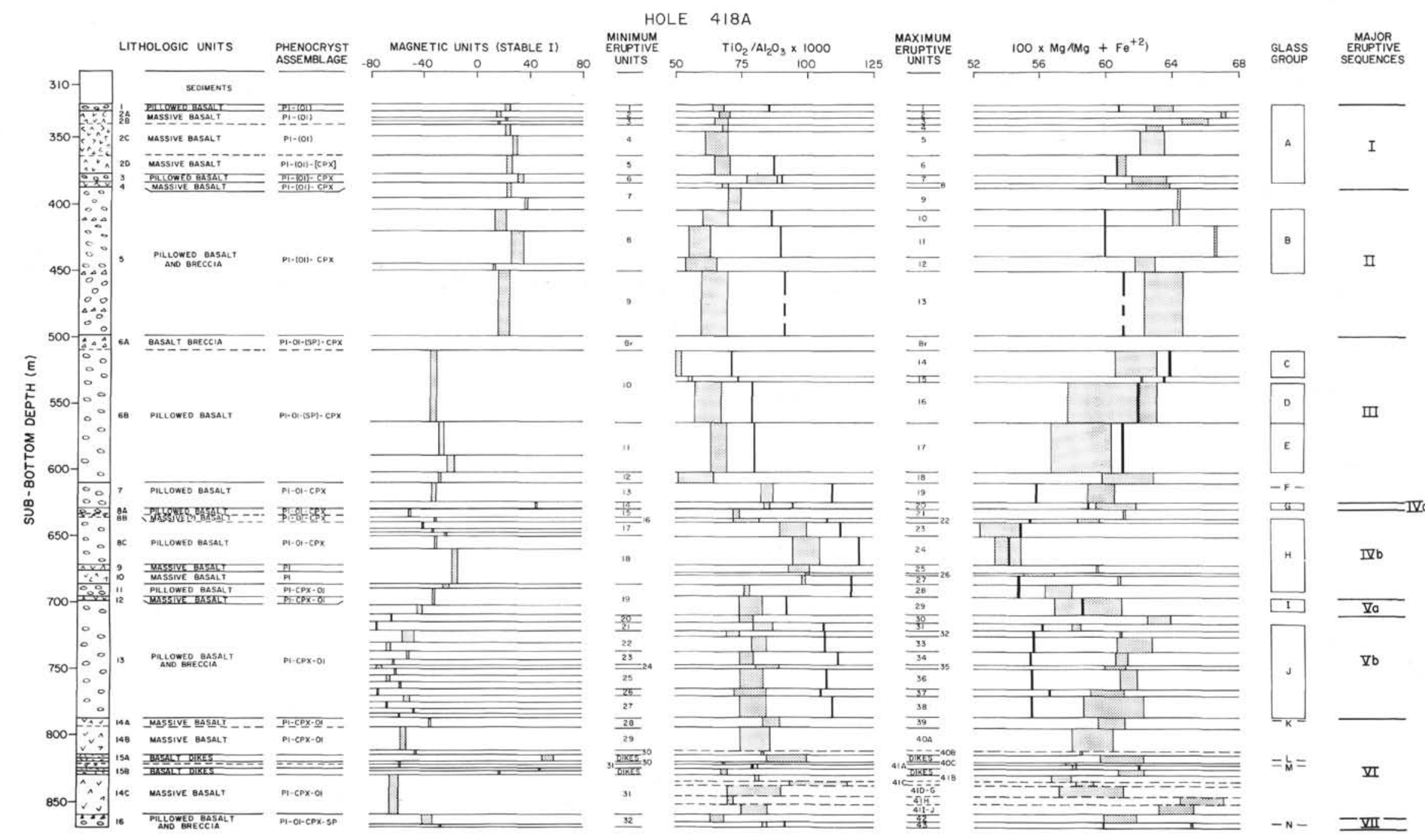

Figure 3. Hole 418A, summary of lithologic, magnetic, and chemical stratigraphy. The lithologic units are updated shipboard core divisions. Magnetic units are based on stable inclination variations measured on board. Eruptive units are interpreted from downhole chemical and magnetic variations; average glass compositions (thick vertical lines) are shown in comparison to the range of whole-rock compositions for each eruptive unit in terms of $\mathrm{TiO}_{2} / \mathrm{Al}_{2} \mathrm{O}_{3}$ and $\mathrm{Mg} /\left(\mathrm{Mg}+\mathrm{Fe}^{+2}\right)$ ratios. Glass compositional groups are after Byerly and Sinton (this volume). 
TABLE 3A

Whole-Rock Compositional Averages for Eruptive Units at Hole 417D, Based on Fresh, Carbonate-Corrected Analyses (see text)

\begin{tabular}{|c|c|c|c|c|c|c|c|c|c|c|c|c|c|c|c|c|c|c|c|c|c|c|c|}
\hline Unit No. & 2 & 5 & 6 & 7 & 8 & 9 & 10 & $\mathrm{BR}^{\mathrm{a}}$ & 11 & 12 & 13 & 14 & 16 & 17 & 18 & 19 & 20 & 21 & 22 & 23 & $24 \mathrm{~A}$ & $24 \mathrm{~B}$ & $x^{b}$ \\
\hline $\mathrm{SiO}_{2}$ & .15 & 0.15 & 50.71 & 50.61 & 50.43 & 0.46 & 49.83 & 50.13 & 49.70 & 50.46 & 50.21 & 50.48 & 50.23 & 50.78 & 51.08 & 50.06 & 50.47 & 50.64 & 49.81 & 50.25 & 50.34 & 50.62 & 49.45 \\
\hline $\mathrm{Al}_{2} \mathrm{O}_{3}$ & 17,10 & 17.52 & 17.54 & 17.78 & 17.60 & 17,32 & 17.40 & 16.72 & 16.93 & 17.67 & 16.01 & 16.04 & 16.64 & 16.62 & 15.43 & 15.53 & 15.98 & 16.39 & 16.63 & 17.13 & 16.32 & 15.37 & 22.52 \\
\hline "FeO" & 9.71 & 9.40 & 9.13 & 9.40 & 9.50 & 9.48 & 9.45 & 9.97 & 9.50 & 8.72 & 10.43 & 10.36 & 10.51 & 9.08 & 10.09 & 10.95 & 10.32 & 9.84 & 10.12 & 9.56 & 9.93 & 10.63 & 7.38 \\
\hline $\mathrm{MgO}$ & & & & & & & 6.7 & & 86 & 6.6 & 6. & 6.65 & 6.58 & 7.02 & 7.02 & 6.67 & 6.41 & 6.57 & 6.95 & 6.12 & 6.84 & 6.94 & 5.32 \\
\hline $\mathrm{CaO}$ & 11.96 & 12.69 & 13.00 & 12.38 & 12.63 & 12.54 & 12.55 & 12.60 & 12.58 & 13.15 & 12.30 & 12.30 & 11.77 & 12.37 & 12.36 & 12.61 & 12.71 & 12.80 & 12,75 & 13.22 & 12.82 & 12.26 & 12.86 \\
\hline $\mathrm{Na}_{2} \mathrm{O}$ & 2.39 & 2.37 & 2.32 & 2.28 & 2.45 & 2.49 & 2.44 & 2.29 & 2.79 & 2.04 & 2.57 & 2.51 & 2.11 & 2.50 & 2.46 & 2.54 & 2.55 & 2.36 & 2.31 & 2.33 & 2.34 & 2.58 & 1.48 \\
\hline $\mathrm{K}_{2} \mathrm{O}$ & 0.07 & 0.06 & 0.04 & 0.13 & 0.18 & 0.09 & 0.06 & 0.04 & 0.06 & 0.03 & 0.07 & 0.13 & 0.21 & 0.07 & 0.06 & 0.07 & 0.04 & 0.02 & 0.05 & 0.02 & 0.03 & 0.04 & 0.06 \\
\hline $\mathrm{TiO}_{2}$ & 1.46 & 1.39 & 1.40 & 1.41 & 1.44 & 1.42 & 1.40 & 1.39 & 1.38 & 1.16 & 1.60 & 1.53 & 1.58 & 1.56 & 1.49 & 1.57 & 1.53 & 1.38 & 1.36 & 1.37 & 1.37 & 1.56 & 0.93 \\
\hline $\mathrm{P}_{2} \mathrm{O}_{5}$ & 0.05 & 0.06 & 0.00 & 0.13 & 0.00 & 0.06 & 0.06 & 0.08 & 0.08 & 0.05 & 0.00 & 0.00 & 0.17 & 0.00 & 0.00 & 0.00 & 0.00 & 0.00 & 0.00 & 0.00 & 0.00 & 0.00 & 0.00 \\
\hline $\mathrm{MnO}$ & 0.06 & 0.06 & 0.00 & 0.11 & 0.00 & 0.06 & 0.06 & 0.08 & 0.11 & 0.05 & 0.00 & 0.00 & 0.20 & 0.00 & 0.00 & 0.00 & 0.00 & 0.00 & 0.00 & 0.00 & 0.00 & 0.00 & 0.00 \\
\hline
\end{tabular}

${ }^{a} \mathrm{BR}=$ breccia.

$\mathbf{b}_{\mathbf{X}}=$ dike.

TABLE 3B

Whole-Rock Compositional Averages for Maximum Eruptive Units at Hole 418A, Based on Fresh, Carbonate-Corrected Analyses (see text)

\begin{tabular}{|c|c|c|c|c|c|c|c|c|c|c|c|c|c|c|c|c|c|c|c|c|}
\hline Unit No. & 1 & 2 & 3 & 4 & 5 & 6 & 7 & 8 & 9 & 10 & 11 & 12 & 13 & $\mathrm{BR}^{\mathrm{a}}$ & 14 & 15 & 16 & 17 & 18 & 19 \\
\hline $\mathrm{SiO}_{2}$ & 50.58 & 51.36 & 50.60 & 50.39 & 50.19 & 50.86 & 50.58 & 50.40 & 50.29 & 50.51 & 50.46 & 49.97 & 49.98 & 49.98 & 49.70 & 49.68 & 49.85 & 49.74 & 49.78 & 50.11 \\
\hline $\mathrm{Al}_{2} \mathrm{O}_{3}$ & 17.34 & 18.24 & 17.83 & 16.72 & 16.95 & 16.49 & 15.88 & 16.85 & 18.52 & 18.84 & 19.79 & 18.14 & 18.15 & 18.61 & 18.46 & 19.38 & 17.63 & 17.97 & 17.98 & 15.85 \\
\hline "FeO" & 8.78 & 7.74 & 8.21 & 9.22 & 8.99 & 9.22 & 9.47 & 9.10 & 8.32 & 7.96 & 7.24 & 8.67 & 8.42 & 8.86 & 8.37 & 7.98 & 9.14 & 9.21 & 8.79 & 10.22 \\
\hline $\mathrm{MgO}$ & 7.36 & 7.33 & 7.15 & 7.41 & 7.22 & 7.12 & 7.64 & 7.09 & 7.11 & 6.70 & 6.60 & 6.81 & 6.80 & 5.52 & 6.39 & 6.12 & 6.74 & 6.15 & 6.67 & 7.32 \\
\hline $\mathrm{CaO}$ & 12.44 & 11.63 & 12.32 & 12.60 & 12.91 & 12.92 & 12.62 & 12.84 & 12.08 & 12.07 & 12.75 & 12.87 & 12.94 & 13.70 & 13.56 & 12.92 & 13.05 & 13.31 & 13.19 & 12.56 \\
\hline $\mathrm{Na}_{2} \mathrm{O}$ & 2.24 & 2.32 & 2.42 & 2.28 & 2.27 & 2.12 & 2.36 & 2.30 & 2.32 & 2.42 & 2.03 & 2.18 & 2.28 & 2.08 & 2.23 & 2.40 & 2.23 & 2.22 & 2.23 & 2.26 \\
\hline $\mathrm{K}_{2} \mathrm{O}$ & 0.05 & 0.09 & 0.12 & 0.07 & 0.09 & 0.05 & 0.04 & 0.10 & 0.07 & 0.15 & 0.06 & 0.08 & 0.06 & 0.16 & 0.08 & 0.18 & 0.08 & 0.06 & 0.05 & 0.04 \\
\hline $\mathrm{TiO}_{2}$ & 1.14 & 1.29 & 1.18 & 1.13 & 1.10 & 1.14 & 1.32 & 1.15 & 1.29 & 1.19 & 1.07 & 1.14 & 1.23 & 1.09 & 0.99 & 1.09 & 1.08 & 1.09 & 0.99 & 1.34 \\
\hline $\mathrm{P}_{2} \mathrm{O}_{5}$ & 0.03 & 0.00 & 0.08 & 0.07 & 0.10 & 0.03 & 0.04 & 0.07 & 0.00 & 0.05 & 0.00 & 0.05 & 0.04 & 0.00 & 0.09 & 0.10 & 0.07 & 0.09 & 0.14 & 0.12 \\
\hline $\mathrm{MnO}$ & 0.04 & 0.00 & 0.08 & 0.11 & 0.17 & 0.05 & 0.05 & 0.09 & 0.00 & 0.11 & 0.00 & 0.08 & 0.08 & 0.00 & 0.13 & 0.15 & 0.13 & 0.15 & 0.17 & 0.18 \\
\hline
\end{tabular}

${ }^{\mathrm{a}} \mathrm{BR}=$ breccia

${ }^{\mathrm{b}} \mathrm{X}$ and $\mathrm{Y}$ are probable dikes.

TABLE 3B - Continued

\begin{tabular}{|c|c|c|c|c|c|c|c|c|c|c|c|c|c|c|c|c|c|c|c|c|}
\hline Unit No. & 20 & 21 & 22 & 23 & 24 & 25 & 26 & 27 & 28 & 29 & 30 & $B R^{a}$ & 31 & 32 & 33 & 34 & 35 & 36 & 37 & 38 \\
\hline $\mathrm{SiO}_{2}$ & 50.17 & 49.62 & 49.69 & 50.17 & 49.21 & 49.63 & 50.38 & 50.03 & 49.01 & 49.51 & 50.19 & 49.64 & 49.52 & 49.61 & 50.06 & 49.65 & 50.52 & 49.89 & 50.03 & 49.97 \\
\hline $\mathrm{Al}_{2} \mathrm{O}_{3}$ & 15.82 & 17.17 & 17.19 & 16.34 & 16.16 & 16.78 & 15.53 & 16.27 & 16.89 & 16.32 & 17.52 & 17.66 & 16.69 & 16.81 & 16.10 & 16.41 & 15.65 & 16.05 & 16.09 & 16.53 \\
\hline "FeO" & 9.79 & 9.19 & 9.46 & 10.40 & 11.11 & 10.21 & 10.88 & 9.94 & 10.37 & 9.98 & 8.59 & 8.99 & 10.03 & 9.60 & 9.85 & 9.78 & 9.95 & 9.84 & 9.88 & 9.64 \\
\hline $\mathrm{MgO}$ & 7.47 & 7.16 & 6.84 & 6.09 & 6.58 & 7.31 & 6.85 & 7.46 & 6.51 & 7.02 & 7.00 & 6.76 & 6.81 & 7.24 & 7.36 & 7.38 & 7.48 & 7.45 & 7.28 & 7.28 \\
\hline $\mathrm{CaO}$ & 12.72 & 12.93 & 12.80 & 12.72 & 12.46 & 11.82 & 11.96 & 11.65 & 12.93 & 13.03 & 12.48 & 12.67 & 12.90 & 12.84 & 12.64 & 12.94 & 12.35 & 12.82 & 12.77 & 12.60 \\
\hline $\mathrm{Na}_{2} \mathrm{O}$ & 2.35 & 2.33 & 2.32 & 2.34 & 2.43 & 2.33 & 2.44 & 2.58 & 2.42 & 2.36 & 2.49 & 2.61 & 2.28 & 2.30 & 2.30 & 2.23 & 2.30 & 2.25 & 2.28 & 2.28 \\
\hline $\mathrm{K}_{2} \mathrm{O}$ & 0.05 & 0.04 & 0.06 & 0.09 & 0.09 & 0.04 & 0.09 & 0.11 & 0.06 & 0.17 & 0.04 & 0.04 & 0.07 & 0.04 & 0.06 & 0.05 & 0.08 & 0.06 & 0.09 & 0.06 \\
\hline $\mathrm{TiO}_{2}$ & 1.32 & 1.26 & 1.35 & 1.49 & 1.61 & 1.58 & 1.56 & 1.60 & 1.48 & 1.30 & 1.34 & 1.34 & 1.37 & 1.25 & 1.30 & 1.25 & 1.34 & 1.30 & 1.28 & 1.30 \\
\hline $\mathrm{P}_{2} \mathrm{O}_{5}$ & 0.13 & 0.13 & 0.13 & 0.20 & 0.16 & 0.14 & 0.13 & 0.16 & 0.14 & 0.14 & 0.18 & 0.14 & 0.13 & 0.13 & 0.13 & 0.13 & 0.13 & 0.13 & 0.13 & 0.13 \\
\hline $\mathrm{MnO}$ & 0.19 & 0.17 & 0.17 & 0.17 & 0.19 & 0.16 & 0.18 & 0.20 & 0.18 & 0.16 & 0.16 & 0.15 & 0.20 & 0.18 & 0.19 & 0.18 & 0.19 & 0.20 & 0.17 & 0.20 \\
\hline
\end{tabular}

TABLE 3B - Continued

\begin{tabular}{|c|c|c|c|c|c|c|c|c|c|c|c|c|c|c|c|c|c|c|c|}
\hline Unit No. & 39 & $40 \mathrm{~A}$ & $40 B$ & $40 \mathrm{C}$ & $41 \mathrm{~A}$ & $41 \mathrm{~B}$ & $41 \mathrm{C}$ & $41 \mathrm{D}$ & $41 \mathrm{E}$ & $41 \mathrm{~F}$ & $41 \mathrm{H}$ & 411 & $41 \mathrm{~J}$ & 42 & 43 & $x^{b} 2$ & $\times 3$ & $\mathrm{Y}^{\mathrm{b}} 1$ & Y3 \\
\hline $\mathrm{SiO}_{2}$ & 50.30 & 50.78 & 50.98 & 50.54 & 50.32 & 50.39 & 50.64 & 50.43 & 50.26 & 49.80 & 49.60 & 50.27 & 49.84 & 49.67 & 50.25 & 50.35 & 50.09 & 49.77 & 49.61 \\
\hline $\mathrm{Al}_{2} \mathrm{O}_{3}$ & 16.36 & 15.98 & 15.92 & 17.05 & 16.52 & 16.42 & 14.39 & 15.51 & 15.97 & 16.72 & 15.92 & 15.87 & 15.79 & 17.90 & 16.07 & 14.68 & 16.05 & 17.26 & 17.57 \\
\hline "FeO" & 9.83 & 9.69 & 9.66 & 9.50 & 9.91 & 9.96 & 10.93 & 10.40 & 9.96 & 9.77 & 9.71 & 9.65 & 9.54 & 8.87 & 9.18 & 11.09 & 9.83 & 9.23 & 9.08 \\
\hline $\mathrm{MgO}$ & 7.22 & 6.76 & 6.57 & 6.30 & 6.67 & 6.56 & 7.64 & 7.37 & 7.37 & 7.21 & 9.04 & 8.10 & 8.40 & 6.70 & 8.24 & 8.09 & 7.44 & 7,08 & 7.02 \\
\hline $\mathrm{CaO}$ & 12.06 & 12.84 & 12.87 & 12.83 & 12.58 & 12.67 & 12.12 & 12.20 & 12.60 & 12.71 & 12.14 & 12.29 & 12.35 & 13.07 & 12.10 & 11.69 & 12.59 & 12.97 & 12.98 \\
\hline $\mathrm{Na}_{2} \mathrm{O}$ & 2.53 & 2.29 & 2.31 & 2.25 & 2.30 & 2.31 & 2.38 & 2.31 & 2.25 & 2.28 & 2.11 & 2.23 & 2.34 & 2.23 & 2.37 & 2.29 & 2.32 & 2.21 & 2.27 \\
\hline $\mathrm{K}_{2} \mathrm{O}$ & 0.07 & 0.05 & 0.03 & 0.04 & 0.05 & 0.04 & 0.03 & 0.07 & 0.03 & 0.04 & 0.06 & 0.04 & 0.10 & 0.07 & 0.13 & 0.03 & 0.04 & 0.03 & 0.03 \\
\hline $\mathrm{TiO}_{2}$ & 1.33 & 1.30 & 1.32 & 1.20 & 1.33 & 1.35 & 1.55 & 1.41 & 1.29 & 1.19 & 1.13 & 1.24 & 1.31 & 1.19 & 1.34 & 1.47 & 1.35 & 1.19 & 1.14 \\
\hline $\mathrm{P}_{2} \mathrm{O}_{5}$ & 0.12 & 0.14 & 0.14 & 0.13 & 0.15 & 0.13 & 0.13 & 0.13 & 0.10 & 0.12 & 0.11 & 0.14 & 0.12 & 0.13 & 0.14 & 0.13 & 0.13 & 0.10 & 0.13 \\
\hline $\mathrm{MnO}$ & 0.18 & 0.17 & 0.20 & 0.16 & 0.17 & 0.17 & 0.19 & 0.18 & 0.17 & 0.16 & 0.17 & 0.17 & 0.20 & 0.18 & 0.17 & 0.19 & 0.17 & 0.16 & 0.17 \\
\hline
\end{tabular}

more pronounced than in Hole 417D, but the greater abundance of chemical and magnetic data for this hole also allows more precise definition of the eruptive history.

In the Hole 418A sequence we recognize seven major eruptive sequences bounded by stratigraphic breaks. Most of the recognized stratigraphic breaks are characterized by zones of higher alteration and three of them are marked by magnetic polarity reversals. Stable magnetic inclination changes occur both at and between the major stratigraphic breaks.

\section{COMPOSITIONAL VARIATIONS OF BASALT MAGMAS}

Byerly and Sinton (this volume) conclude from glass compositional variation that shallow-level crystal fractionation accounts for most observed variations in liquid compo- 
sitions, but that multiple parent liquids may be required to account for the total variation. We are not yet able to give a definitive account of fractionation relationships, since this requires comprehensive treatment of our data. However, we can use average compositions for each chemical unit to summarize variation features and to point to the most likely fractionation processes.

Figures $4 \mathrm{a}, \mathrm{b}$ and $5 \mathrm{a}$, b show average (corrected) compositions of each eruptive unit plotted in terms of $\mathrm{MgO}$ and $\mathrm{Al}_{2} \mathrm{O}_{3}$ versus $\mathrm{TiO}_{2}$. Each composition is classified according to the glass group defined by Byerly and Sinton (this volume) with which they are associated in the core. Wholerock compositions clearly do not follow a single fractionation path and if, as appears likely, the glasses are restricted
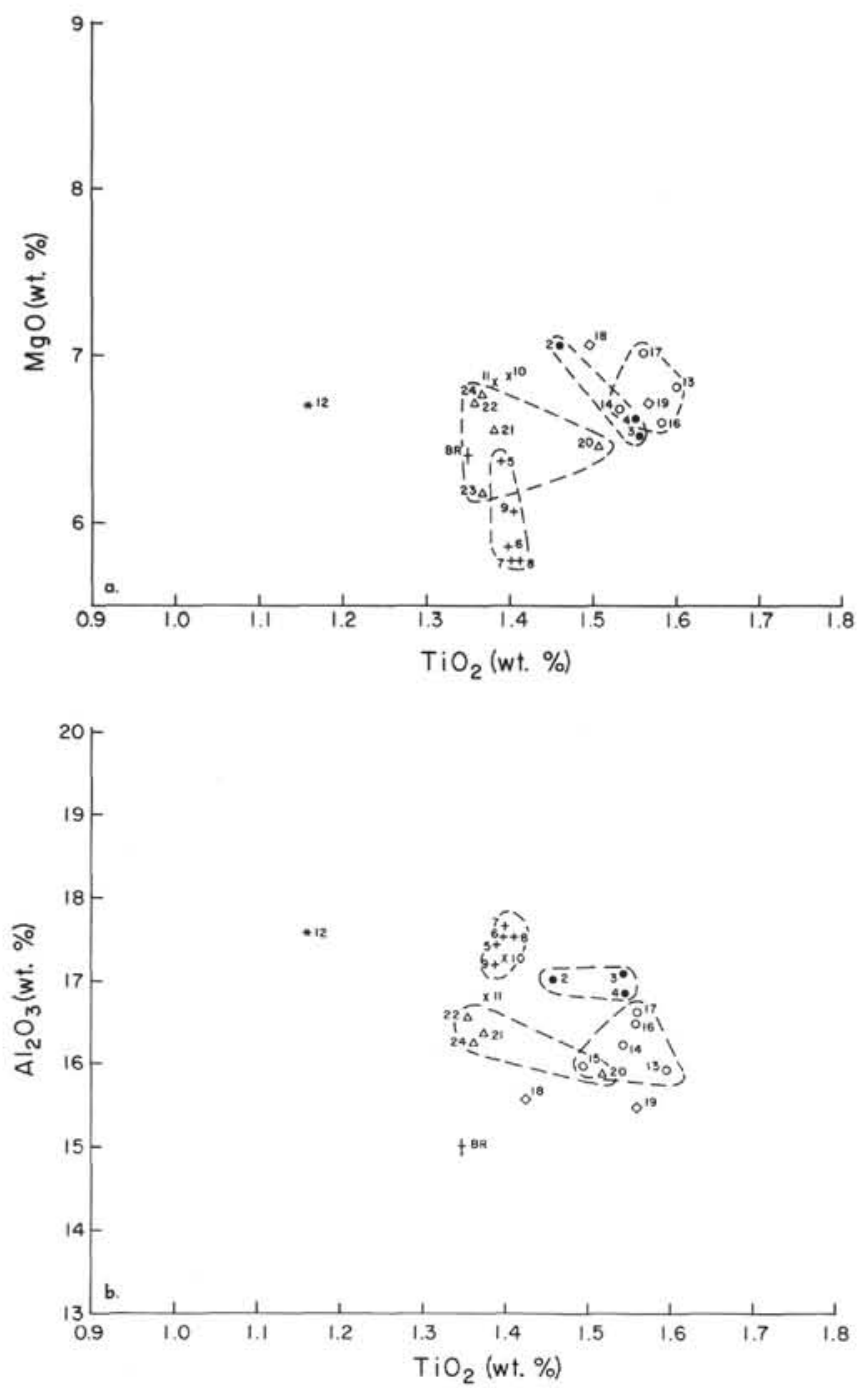

Figure 4. $\mathrm{MgO}$ and $\mathrm{Al}_{2} \mathrm{O}_{3}$ versus $\mathrm{TiO}_{2}$ for Hole 417D; (a) $\mathrm{MgO}$ versus $\mathrm{TiO}$, and (b) $\mathrm{Al}_{2} \mathrm{O}_{3}$ versus $\mathrm{TiO}_{2}$. Whole-rock average compositions for maximum eruptives units are identified according to the associated glass compositional groups (Byerly and Sinton, this volume). Glass group symbols: Hole 417D -A ; - B +; -C $x ;-D * ;-E ~ o ;-F \diamond$; $-G+;-H \triangle$. Units 1 and 15 are defined on glass compositions alone.
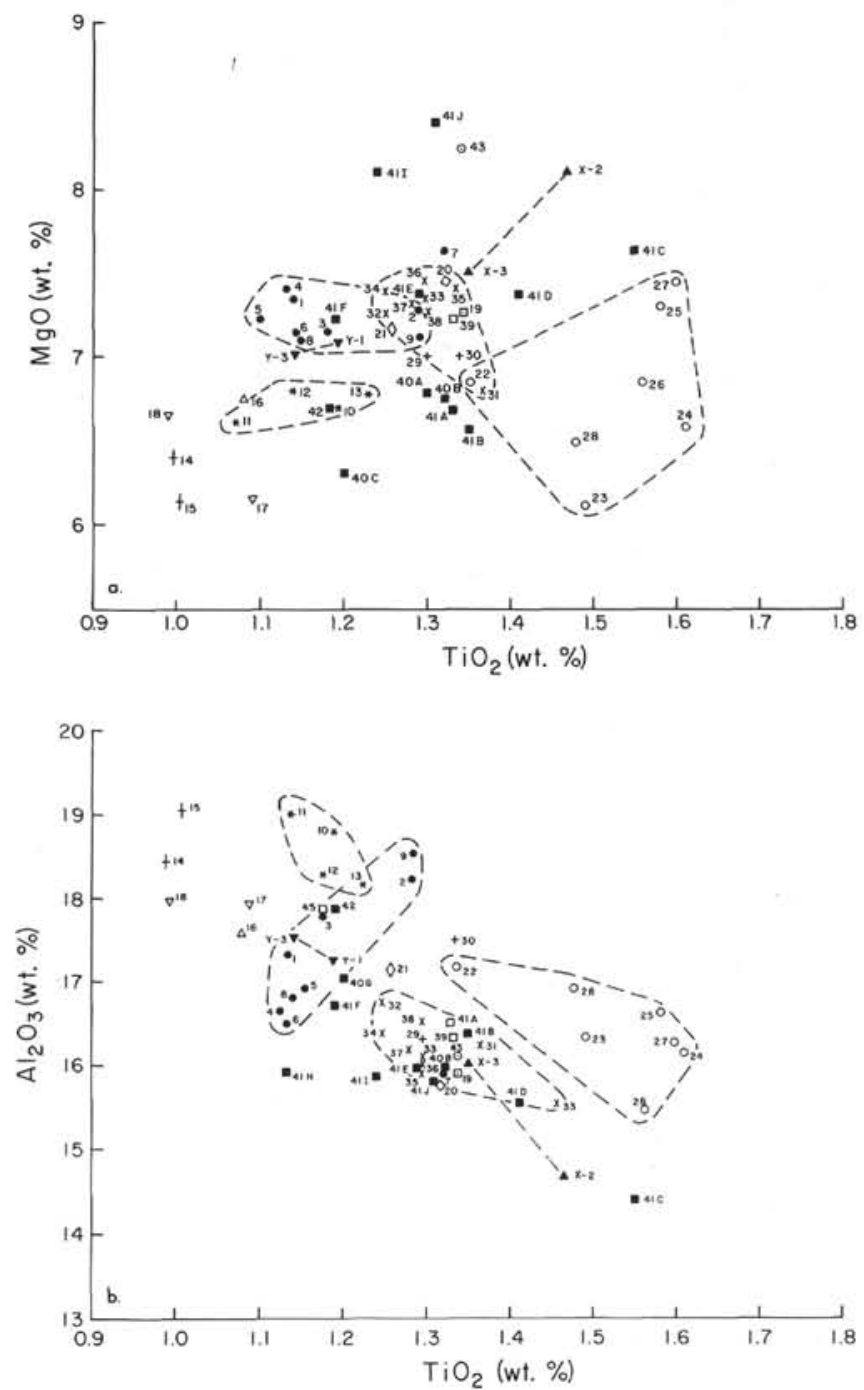

Figure 5. $\mathrm{MgO}$ and $\mathrm{Al}_{2} \mathrm{O}_{3}$ versus $\mathrm{TiO}_{2}$ for Hole 418A; (a) $\mathrm{MgO}$ versus $\mathrm{TiO}_{2}$, and (b) $\mathrm{Al}_{2} \mathrm{O}_{3}$ versus $\mathrm{TiO}_{2}$. Average compositions for maximum eruptive units are identified according to glass compositional groups (Byerly and Sinton, this volume). Glass group symbols: Hole 418A -A •; $-B$ *; $-C \dagger ;-D \triangle ;-E \triangle ;-F \square ;-G \diamond ;-H o ;-I+;-J x ;-K$ 口; -L (dike, $x$-type $\mathbf{\Delta}) ;-L$ (dike, $y$-type $\mathbf{\Delta}) ;-M=$ ); $-N$ o.

to a single line of liquid descent, whole-rock variation must result from differential phenocryst movement during and after a major crystallization stage at depth. The variation pattern thus reflects the unique modal mineralogy and fractionation history of any single eruption.

The comparatively low values of $\mathrm{Mg} /\left(\mathrm{Mg}+\mathrm{Fe}^{+2}\right)(0.50$ to 0.65$)$ and $\mathrm{TiO}_{2} / \mathrm{Al}_{2} \mathrm{O}_{3}(0.070$ to 0.120$)$ in aphyric and sparsely phyric samples and the complex phenocryst assemblages of phyric basalts indicate that all of the lavas are evolved compared to primitive mantle melts. The liquid (glass) fraction conforms to a low-pressure three-phase cotectic crystallization trend (Byerly and Sinton, this volume) typical of ocean-floor tholeiite crustal fractionation.

Figures 2 and 3 show the downhole variations of wholerock and associated glass compositions for individual eruptive units. $\mathrm{TiO}_{2} / \mathrm{Al}_{2} \mathrm{O}_{3}$ variation shows the widespread ef- 
fects of accumulated plagioclase in lavas at both sites. An exception appears to be lithologic Unit 14c in Hole 418A, a massive aphyric to sparsely phyric flow in which considerable internal fractionation seems to have occurred during and after outflow (Figure 3), with only few portions of the flow enriched in plagioclase.

In contrast to plagioclase, olivine and in some cases, early formed clinopyroxene, appear to have been in equilibrium or nearly so with the associated liquids $\left(\mathrm{KD}_{\mathrm{D}}=\mathrm{XFeO}^{\circ 1}\right.$

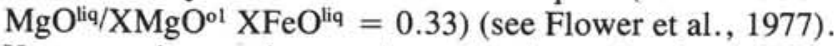
However, in certain eruptive units (e.g., Sections 417D$10-13,418 \mathrm{~A}-15-18$, and 418A-45-46) early formed clinopyroxene appears to coexist metastably with spinel and shows a reaction relation with the liquid phase.

The discrepancy in $\mathrm{Mg} /\left(\mathrm{Mg}+\mathrm{Fe}^{+2}\right)$ ratios for wholerock and glass compositions (Figures 2 and 3) suggests a more complex role for mafic phases during fractionation than for plagioclase. Whereas $\mathrm{TiO}_{2} / \mathrm{Al}_{2} \mathrm{O}_{3}$ ratios for wholerock compositions are mostly lower than those of associated glasses, whole-rock $\mathrm{Mg} /\left(\mathrm{Mg}+\mathrm{Fe}^{+2}\right)$ ratios may be greater, less than, or equal to the glass values (Figures 2 and 3 ). These relationships suggest some degree of decoupling between mafic and feldspathic crystals, and possibly between olivine and clinopyroxene crystals, during the fractionation process. Another possible explanation is the development of iron-rich smectite in the crystalline rocks (cf., Byerly and Wright, in press). Although a majority of eruptive units at both sites show higher $\mathrm{Mg} /\left(\mathrm{Mg}+\mathrm{Fe}^{+2}\right)$ ratios for wholerock compositions than for glass, there are several notable exceptions (e.g., units 15 through 18 in Hole 418A).

Because any single glass compositional group may include more than one eruptive unit, we assume that differences in liquid-fraction composition of magmas are largely the result of pre-eruption fractionation. Phenocrysts appear to be exclusively of low-pressure origin, so that fractionation probably comprised protracted crystallization under relatively steady conditions coupled with gravity separation of the crystal-liquid fractions. In theory, if eruption rates are sufficiently high, so that no further crystallization occurs during eruption, the liquid fraction should be in equilibrium with the pre-eruptive crystalline phases. Further crystallization of phenocrysts or microphenocrysts after eruption would coincide with rapid chilling of the liquid fraction, resulting in negligible compositional changes. Hence, in either case, glass compositions should be relatively uniform over discrete core intervals, assuming that the magma chamber was not compositionally zoned or that mixing of fractionated and primitive magmas did not occur during eruption. Phenocryst redistribution during and after eruption probably accounts for much of the discrepancy between glass and whole-rock compositions, especially where whole-rock $\mathrm{Mg} /\left(\mathrm{Mg}+\mathrm{Fe}^{+2}\right)$ ratios exceed those of glass. However, the widespread occurrence of accumulated plagioclase suggests this is the result of temporary magma arrest at depth in reservoirs permitting massive flotation of this phase.

Trace-element studies generally confirm the findings from major elements. Plots of alteration-resistant lithophile elements $\mathrm{Zr}, \mathrm{Y}$, and $\mathrm{Ti}$ are shown in Figures $6 \mathrm{a}$ and $6 \mathrm{~b}$ as average values for Hole $418 \mathrm{~A}$ eruptive units, identified according to the associated glass groups (Byerly and Sinton,
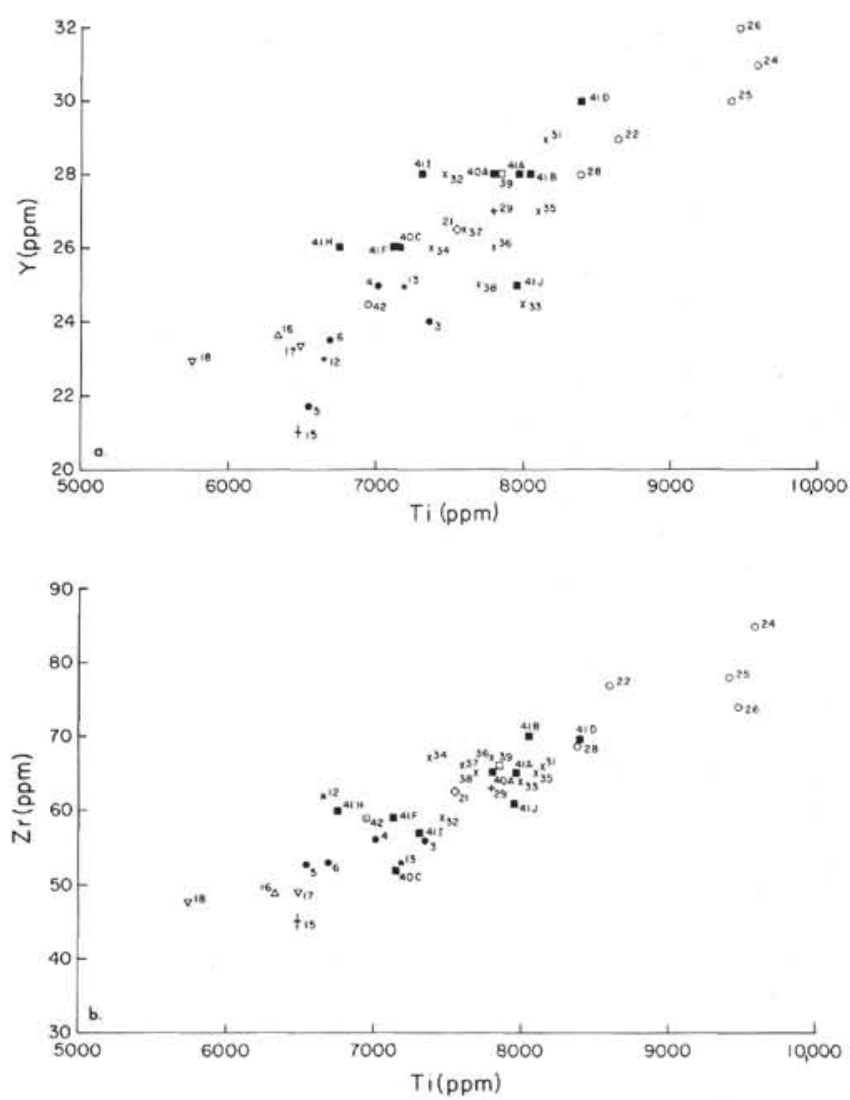

Figure 6. $Y$ and $Z r$ versus Ti for Hole 418A; (a) $Y$ versus $T i$ (ppm), and (b) $\mathrm{Zr}$ versus $\mathrm{Ti}(\mathrm{ppm})$. Average compositions for maximum eruptive units are identified according to glass compositional groups; symbols as in Figure 5 .

this volume). $\mathrm{Zr}$ and $\mathrm{Y}$ versus $\mathrm{Ti}$ exhibit positive linear patterns of essentially constant interelement ratios $(\mathrm{Zr} / \mathrm{Y}=$ 2.1 to $2.4 ; \mathrm{Ti} / \mathrm{Y}=273$ to $297 ; \mathrm{Ti} / \mathrm{Zr}=121$ to 133 ) and show no significant differences between chemical groups. Differences in abundance of these elements can probably be attributed to fractional crystallization and/or crystal accumulation, because enrichment factors (1.5 to 1.6) are no greater than would be expected for the range of major element variation. Multiple primary magma batches would be required if the variation could not be modeled by fractional crystallization of the observed (or postulated) liquidus phases. As a tentative conclusion we suggest that parental magmas at Holes 417D and 418A were similar in composition, but may nonetheless have shown subtle differences between consecutive batches.

\section{STRATIGRAPHIC SUMMARY}

Stratigraphic sections at both Holes 417D and 418A show sharp chemical and lithologic discontinuities accompanied by changes in stable magnetic inclination and sometimes in polarity. However, core intervals between major discontinuities also often show significant secular changes in both basalt compositions and stable magnetic inclinations (Figures 2 and 3). Good examples are eruptive sequences I, II, IV, and V in Hole 418A and sequences II and III in Hole 417D. Some of these intervals show an upward transition 
from evolved to less-evolved compositions corresponding in certain cases to progressive changes in stable inclinations. Such patterns appear to reflect cyclic activity during crustal formation. A single cycle appears to comprise a sequence of several eruptions from the same conduit system. Succeeding cycles may tap completely new conduit systems depending on the duration of the preceding quiescent period and the crustal spreading rate (see Flower et al., 1977). The pattern of magnetic inclinations observed may thus reflect a systematic regime of tectonic deformation accompanying eruptive episodes, and indicate subsidence due to lateral spreading and the exhaustion of temporary magma reservoirs. If this interpretation is correct, inclinations of the uppermost flows in a newly active cycle should show the smallest departure from dipole values assuming deposition was horizontal. Initiation of a new cycle from a separate system should result in nonconformable deposition of new lavas and further (although to a progressively lesser extent) tilting of the underlying pile.

The downhole magnetic-inclination pattern transgresses the dipole average value seven times in Hole 418A and four times in Hole 417D, showing that "aberrant"' stable inclinations are not exclusively high or low. This may indicate oscillatory movement of the spreading axis in which crustal build-up proceeds with sequential eruptive episodes and interim periods of tilting with lateral movement whose direction is only established unambiguously on final completion of the pile. Such a process is consistent with the decrease in variability of inclination towards the top of Holes 418A and 417D, although in the latter the inclinations are consistently higher than dipole values (Figures 2 and 3). In either case, the lithologic, chemical, and magnetic stratigraphy becomes simpler as this segment of crust evidently became finally committed to the western spreading limb.

The alteration profiles at Holes 417D and 418A provide further evidence for episodic eruption. A strong repetitive downhole pattern is shown by $\mathrm{K}_{2} \mathrm{O}$, and to a lesser extent, by $\mathrm{H}_{2} \mathrm{O}^{+}$, reflecting mainly oxidative alteration to produce $\mathrm{K}$-rich smectite. This is most concentrated at and below depositional surfaces of "exposed" lava sequences delineated by the major stratigraphic breaks (Figures 7a and 7b). Above many of these horizons a comparatively sharp drop occurs in $\mathrm{K}_{2} \mathrm{O}$ and $\mathrm{H}_{2} \mathrm{O}^{+}$. Minor variants of this pattern are also present, suggesting several lesser gaps in the eruptive record.

In both sections the episodic pattern is superimposed on an overall downhole decrease in oxidation and $\mathrm{H}_{2} \mathrm{O}^{+}$content based on data from flow interiors and from lower, less altered, parts of individual cyclic intervals (Figures 7a and 7b). This "'pervasive"' alteration gradient appears to terminate at a depth of about 850 meters, where contents of $\mathrm{H}_{2} \mathrm{O}^{+}$ and $\mathrm{K}_{2} \mathrm{O}$ are effectively juvenile. The increasing freshness with depth of drilled basement - especially of glass and groundmass minerals such as olivine - was one of the major surprises resulting from drilling of Cretaceous crust. Presumably, typical crust of this age is mostly fresh below about 500 meters sub-basement depth, and is altered only during short-lived periods of exposure to sea water between eruptive episodes and prior to sediment deposition. On the assumption that time gaps between major eruptive sequences are of the same approximate length, the downhole decrease in alteration must reflect a sharp decline in circula-
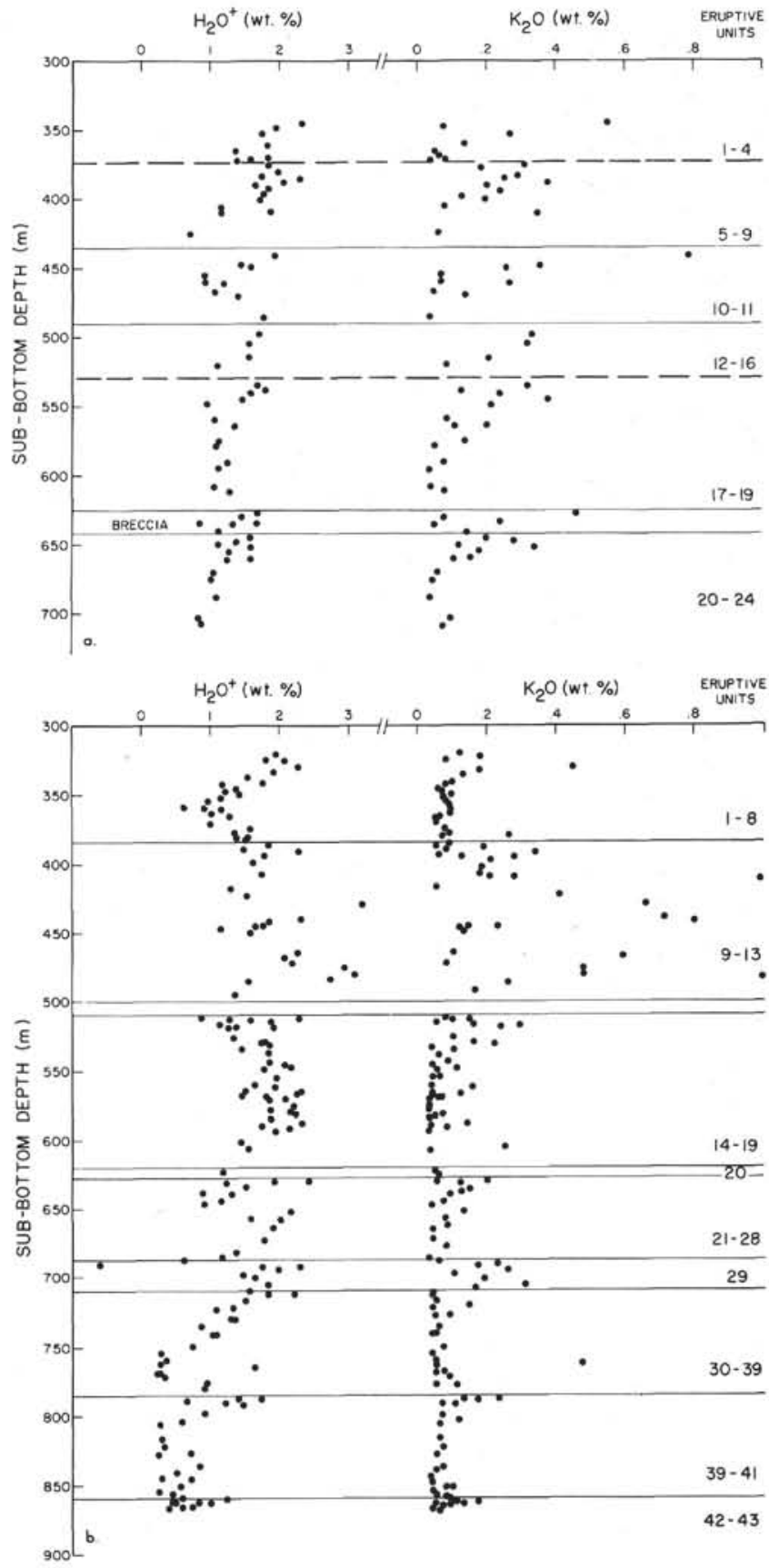

Figure 7. Plots of $\mathrm{H}_{2} \mathrm{O}$ and $\mathrm{K}_{2} \mathrm{O}$ versus sub-bottom depth. Horizontal lines show major stratigraphic breaks, dashed lines subsidiary breaks; (a) Hole 417D, and (b) Hole $418 \mathrm{~A}$.

tion of sea water through the basaltic crust as it moves away from the spreading axis. We conclude that crust at Holes 417D and 418A (but not Hole 417A) was effectively sealed soon after construction, possibly by sedimentation, and that circulating water was progressively restricted to shallower levels as its availability declined and hydrous alteration phases formed in the pile.

\section{CONCLUSIONS}

In contrast to previous attempts at basement drilling in the Atlantic Ocean, deep penetration and excellent recovery at Sites 417 and 418 allow detailed reconstruction of the erup- 
tive stratigraphy. The main conclusions from study of Holes 417D and 418A may be summarized as follows:

1) Eruptive crust was probably built up by a number of small eruptions, each characterized by flows of relatively uniform composition and stable magnetic inclinations.

2) At both sites larger scale eruptive cycles apparently reflect draining of separate high-level magma chambers. Generally, the boundaries between these large cycles are marked by lithologic, chemical, and magnetic breaks in the core.

3) The resulting pattern of chemical stratigraphy is matched by variable stable magnetic inclinations suggesting that eruptive episodes undergo progressive tectonic deformation, possibly in the context of an oscillating spreading axis.

4) Downhole alteration profiles reflect rapid crustal sealing, probably by sedimentation and growth of secondary phases, causing reduced permeability. Zones of intense oxidative alteration coincide generally with noneruptive periods.

5) In contrast to the modern Mid-Atlantic Ridge at $22^{\circ} \mathrm{N}$ and $37^{\circ} \mathrm{N}$, the inferred chemistry of primary magma batches at Sites 417 and 418 reflects comparatively uniform conditions of partial melting and source composition.

6) Compositional variations in the erupted basalts are probably due largely to crystal fractionation and posteruption phenocryst redistribution.

\section{ACKNOWLEDGMENTS}

The manuscript was read critically by Tom L. Wright.

\section{REFERENCES}

Abbey, S., 1973. Studies in "standard samples" of silicate rocks and minerals. Part 3. 1973 extension and revision of "usable" tables, Canadian Geol. Publ., p. 1-24.

Byerly, G. and Wright, T. L., in press. Origin of major element chemical trends in DSDP Leg 37 basalts from the Mid-Atlantic Ridge, J. Volcanol. Geotherm. Res.

Flower, M. F. J., Robinson, P. T., Ohnmacht, W., and Schmincke, H.-U., 1977. Magma fractionation systems beneath the Mid-Atlantic Ridge, $36-37^{\circ} \mathrm{N}$, Contrib. Mineral. Petrol., v. 64, p. 167-195.

Flower, M. F. J., Schmincke, H.-U., Robinson, P. T., Ohnmacht, W., Parker, R., and Gibson, I. L., 1978. Petrology and chemistry of eruptive rocks, DSDP Leg 46. In Dmitriev, L., Heirtzler, J., et al., Initial Reports of the Deep Sea Drilling Project, v. 46: Washington (U.S. Government Printing Office), p. $425-445$.

Hall, J. M., in press. The magnetic properties and magnetization of oceanic basalts and the implications for the history and structure of oceanic Layer 2, Second Maurice Ewing Symposium, AGU special volume.

Watkins, N. and Walker, G. F., 1977. Magnetostratigraphy of eastern Iceland, Am. J. Sci., v. 277, p. 513-584. 